\title{
Late Permian to Middle Triassic palaeogeographic differentiation of key ammonoid groups: evidence from the former USSR
}

\author{
Yuri D. Zakharov¹, Alexander M. Popov¹ \& Alexander S. Biakov² \\ 1 Far-Eastern Geological Institute, Russian Academy of Sciences (Far Eastern Branch), Stoletija Prospect 159, Vladivostok, RU-690022, Russia \\ 2 North-East Interdisciplinary Scientific Research Institute, Russian Academy of Sciences (Far Eastern Branch), Portovaja 16, Magadan, RU-685000, \\ Russia
}

\begin{abstract}
Keywords
Ammonoids; palaeobiogeography;

palaeoclimatology; Permian; Triassic.

\section{Correspondence}

Yuri D. Zakharov, Far-Eastern Geological

Institute, Russian Academy of Sciences (Far

Eastern Branch), Vladivostok, RU-690022,

Russia. E-mail: yurizakh@mail.ru
\end{abstract}

doi:10.1111/j.1751-8369.2008.00079.x

\begin{abstract}
Palaeontological characteristics of the Upper Permian and upper Olenekian to lowermost Anisian sequences in the Tethys and the Boreal realm are reviewed in the context of global correlation. Data from key Wuchiapingian and Changhsingian sections in Transcaucasia, Lower and Middle Triassic sections in the Verkhoyansk area, Arctic Siberia, the southern Far East (South Primorye and Kitakami) and Mangyshlak (Kazakhstan) are examined. Dominant groups of ammonoids are shown for these different regions. Through correlation, it is suggested that significant thermal maxima (recognized using geochemical, palaeozoogeographical and palaeoecological data) existed during the late Kungurian, early Wuchiapingian, latest Changhsingian, middle Olenekian and earliest Anisian periods. Successive expansions and reductions of the warmtemperate climatic zones into middle and high latitudes during the Late Permian and the Early and Middle Triassic are a result of strong climatic fluctuations.
\end{abstract}

Prime Middle-Upper Permian, Lower and Middle Triassic sections in the former USSR and adjacent territories are currently located in Transcaucasia (Ševyrev 1968; Kotljar et al. 1983; Zaharov 1985; Zakharov, Biakov et al. 2005), North Caucasus (Kotljar et al. 1983; Kotlyar et al. 2004; Zakharov 1986), Mangyshlak (Astahova 1960; Ševyrev 1968, 2002; Gavrilova 1980, 1989; Balini et al. 2000), Verkhoyansk area (Zaharov 1971; Dagys \& Ermakova 1996; Zakharov 2002), Arctic Siberia (Mojsisovics 1886; Lazurkin \& Korčinskaja 1963; Zaharov 1978; Dagis \& Ermakova 1988; Dagys \& Ermakova 1996), Spitsbergen (Mørk et al. 1999; Worsley 2006) and the Far East (Russian Far East and Kitakami; Burij 1959; Kiparisova 1961, 1972; Zaharov 1968, 1978; Zaharov \& Pavlov 1986a, b; Zakharov 1992, 1996, 1997; Zakharov \& Oleinikov 1994; Okuneva 1976; Ehiro \& Bando 1985; Ehiro et al. 1986; Ehiro 1995, $2001 \mathrm{la,} \mathrm{b;} \mathrm{Ehiro} \mathrm{\&} \mathrm{Araki} \mathrm{1997;}$ Ehiro \& Misaki 2005). The first reports on PermianTriassic ammonoids from these areas were published by Buch (1831) (Bogdo Mount, Russian Platform), Keyserling (1845) (Arctic Siberia), Abich (1878) (Transcaucasia), Diener (1895b) (Russian southern Far East),
Bajarunas (1936) (Mangyshlak and Kazakhstan), Popov (1939, 1958) (Russian northern Far East and Verkhoyansk area) and Kiparisova (in Voinova et al. 1947) (North Caucasus). However, our knowledge of Permian-Middle Triassic ammonoids and palaeobiogeography, and especially of the Olenekian-Anisian boundary, remains limited (Diener 1916; Zakharov 1974; Zaharov 1977; Dagys 1988; Ehiro 1997; McGowan 2005; Brayard et al. 2006; Brayard et al. 2007; Brühwiler et al. 2007; Galfetti, Hochuli et al. 2007; Brayard \& Bucher 2008; Brayard et al. in press).

The purpose of this study is the analysis of diversity patterns of Permian-Early Anisian ammonoids from the former USSR area and neighbouring territories, for palaeoecological and palaeogeographical reconstructions.

\section{Materials}

Original palaeontological materials used for our investigation were obtained from the Permian of Transcaucasia, South Primorye, Kitakami (Japan) and from 


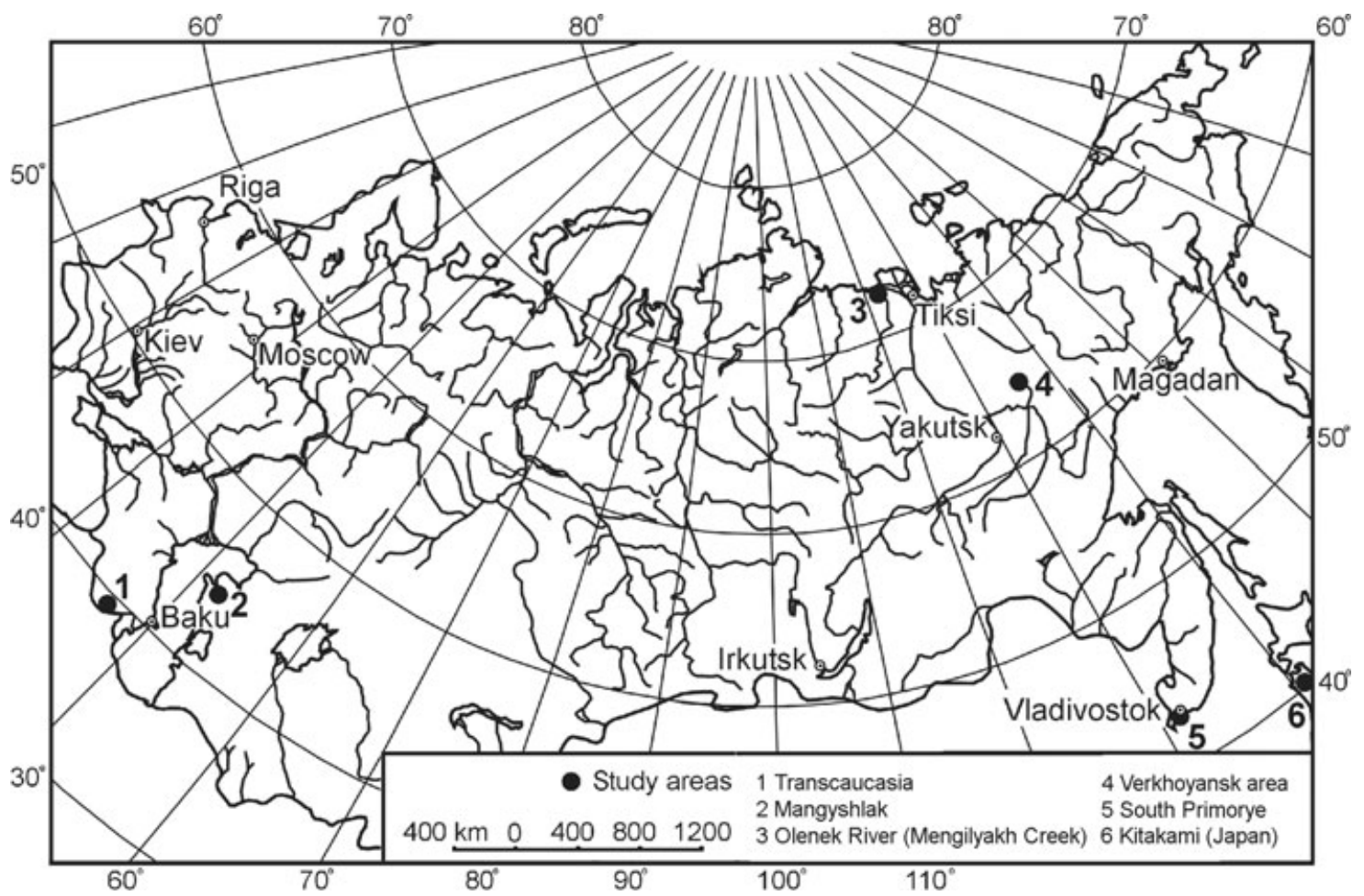

Fig. 1 Location map of sections sampled in the former USSR and Kitakami (Japan).

the Lower-Middle Triassic of the Verkhoyansk area, Arctic Siberia, South Primorye and Mangyshlak (Fig. 1).

\section{Ammonoid successions}

Within the Permian-Triassic ammonoid successions examined, some consecutive phases can be distinguished. It has been been proposed to call them megaclimaxes, confinis and pioneer phases (Zakharov 1983). The designation "megaclimaxes" (descended from Clements's [1916, 1936] biological term "climax") was proposed for the phases during which many or basic assemblage elements acquired a high diversity, and it was proposed that the phases between megaclimaxes be called confinis (from the Latin confinis, meaning adjacent). The confinis phases corresponding to the invasions of the first settlers can be designated as pioneer (or initial) ones.

\section{Permian of the Far East (South Primorye and Kitakami)}

The generalized stratigraphical distribution of Permian ammonoids from South Primoye and Kitakami are presented by Zakharov \& Ehiro (in press). Among Permian cephalopods, cyclolobid ammonoids seem to be the most important group for phylogenetic and palaeogeographic reconstructions, because of their morphological characteristics. Specifically, they possess the most progressive suture lines among the Goniatitida, and have a wide geographical distribution. The most commonly occurring genera of cyclolobid ammonoids for the Middle and Upper Permian are Timorites and Cyclolobus, respectively, although some latest Timorites are also known in the lower Wuchiapingian (Ehiro \& Araki 1997; Zakharov \& Ehiro in press).

\section{Wuchiapingian-Lower Induan of Transcaucasia}

In the Late Permian ammonoid succession of Transcaucasia, the early Wuchiapingian and late Changhsingian possessed the most diversified and abundant ammonoid occurrences (Zakharov, Biakov et al. 2005) (Fig. 2). Goniatite Pseudogastrioceras abichianum (Möller) and ceratite Paratirolites vediensis Shevyrev ammonoids became dominant during the early Wuchiapingian and late Changhsingian, respectively.

\section{Permian-Triassic boundary beds in the Verkhoyansk area}

The Setorym River is the most important locality containing the Otoceras fauna in the Verkhoyansk area (Zaharov 


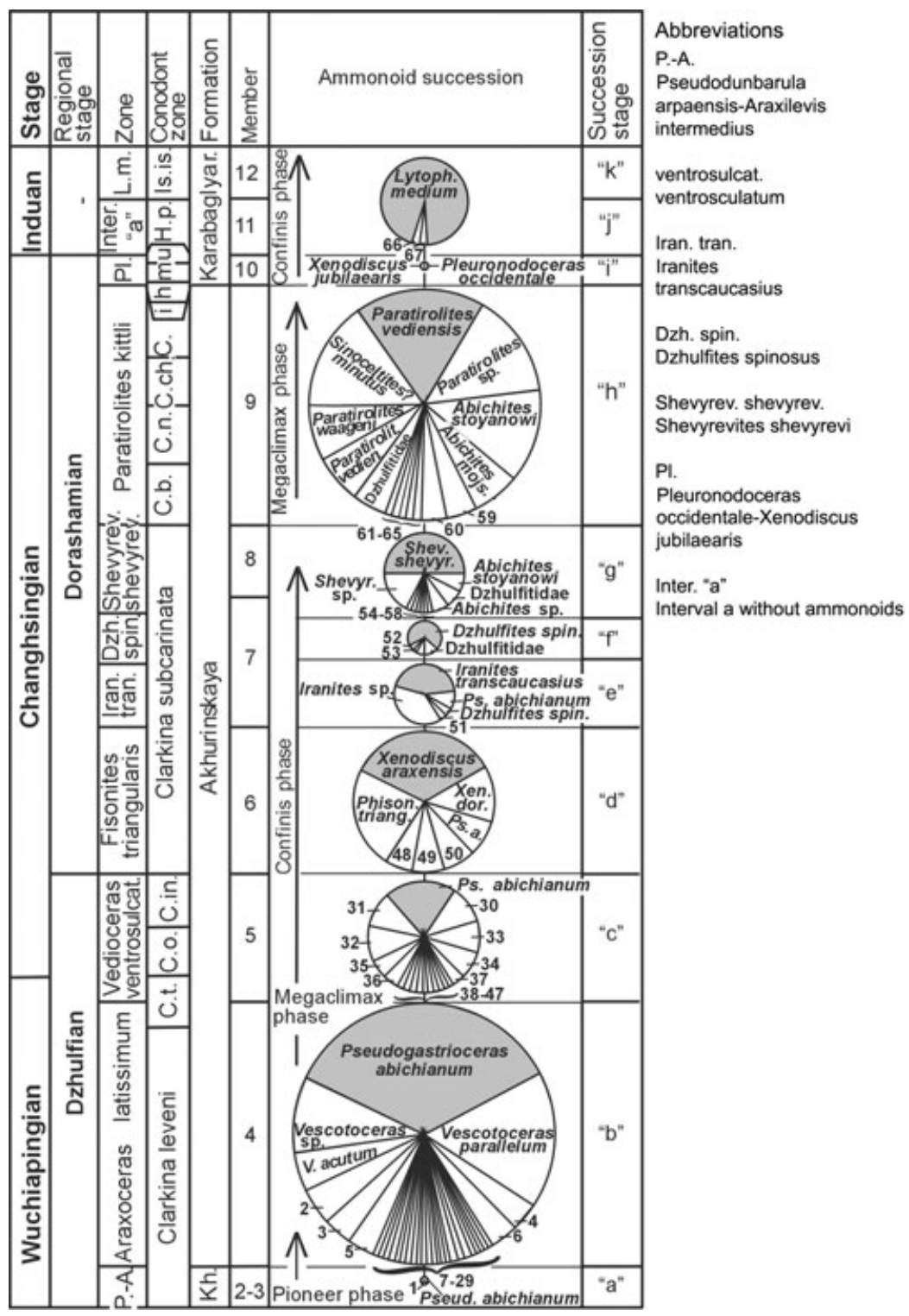

Fig. 2 Change of species composition and proportion in the Late Permian ammonoid succession of Transcaucasia: 1, Vescotoceras parallelum Ruzhencev; 2, Prototoceras tropitum (Abich); 3, Vescotoceras serratum Ruzhencev; 4, Vescotoceras evanidum Ruzhencev; 5, Vescotoceras pessoides (Abich); 6, Araxoceras latissimum Ruzhencev; 7-29, others, including Kingoceras achurense Zakharov, Eumedlicottia stoyanowi (Nassichuk, Furnish \& Glenister), Strigogoniatites sp., Basleoceras kotljarae Zakharov, Araxoceras latum Ruzhencev, Araxoceras varicatum Ruzhencev, Araxoceras glenisteri Ruzhencev, Araxoceras rotoides Ruzhencev, Araxoceras tectum Ruzhencev, Araxoceras trochoides (Abich), Araxoceras sp., Rotaraxoceras caucasium Ruzhencev, Rotaraxoceras sp., Prototoceras intermedium (Abich), Prototoceras discoidale Ruzhencev, Prototoceras raddei (Arthaber), Prototoceras fedoroffi (Arthaber), Prototoceras sp., Changhsingoceras ruzhencevi (Zakharov), Urartoceras abichianum Ruzhencev, Avushoceras sp., Pseudotoceras djoulfense (Abich), Pseudotoceras sp.; 30, Avushoceras jakovlewi Ruzhencev; 31, Vedioceras ogbinense Ruzhencev; 32, Vedioceras umbonovarum Ruzhencev; 33, Vedioceras sp.; 34, Vedioceras ventroplanum Ruzhencev; 3, Vedioceras ventrosulcatum Ruzhencev; 36, Dzhulfoceras fürnishi Ruzhencev; 37, Avushoceras sp.; 38-47, others, including Urartoceras abichianum Ruzhencev, Prototoceras tropitum (Abich), Pseudotoceras admirabile Rostovcev and Azarian, Rotaraxoceras deruptum Ruzhencev, Dzhulfotoceras paulum Ruzhencev, Dzhulfotoceras inflatum Ruzhencev, Changhsingoceras ruzhencevi (Zakharov), Pseudotoceras sp., Pseudotoceras djoulfense (Abich), Pseudotoceras armeorum Ruzhencev; 48, Stacheoceras tschernyschewi (Stoyanow); 49, Phisonites? sp.; 50, Xenodiscus sp.; 51, Xenodiscus sp.; 52, Stacheoceras tschernyschewi (Stoyanow); 53, Pseudogastrioceras abichianum (Möller); 54-58, others, including Pseudogastrioceras abichianum (Möller), Dzhulfites spinosus Shevyrev, Paratirolites waageni (Stoyanow), Paratirolites sp., Abichites abichi (Shevyrev); 59, Paratirolites kittli Stoyanow; 60, Abichites sp.; 61-65, others, including Pseudogastrioceras abichianum (Möller), Abichites abichi Shevyrev, Paratirolites trapezoidalis Shevyrev, Paratirolites dieneri Stoyanow, Pseudotirolites? azariani Rostovcev; 66, Lytophiceras sp.; 67, Ophiceratidae gen. et sp. indet. The size of the circle plot in this and subsequent figures is proportional to the abundance of ammonoid taxa in a succession stage. Observations were from 390 (stage b), 170 (stage c), 210 (stage d), 90 (stage e), 50 (stage f), 75 (stage g), 340 (stage h) and 130 (stages $\mathrm{j}$ and k) samples, respectively. 


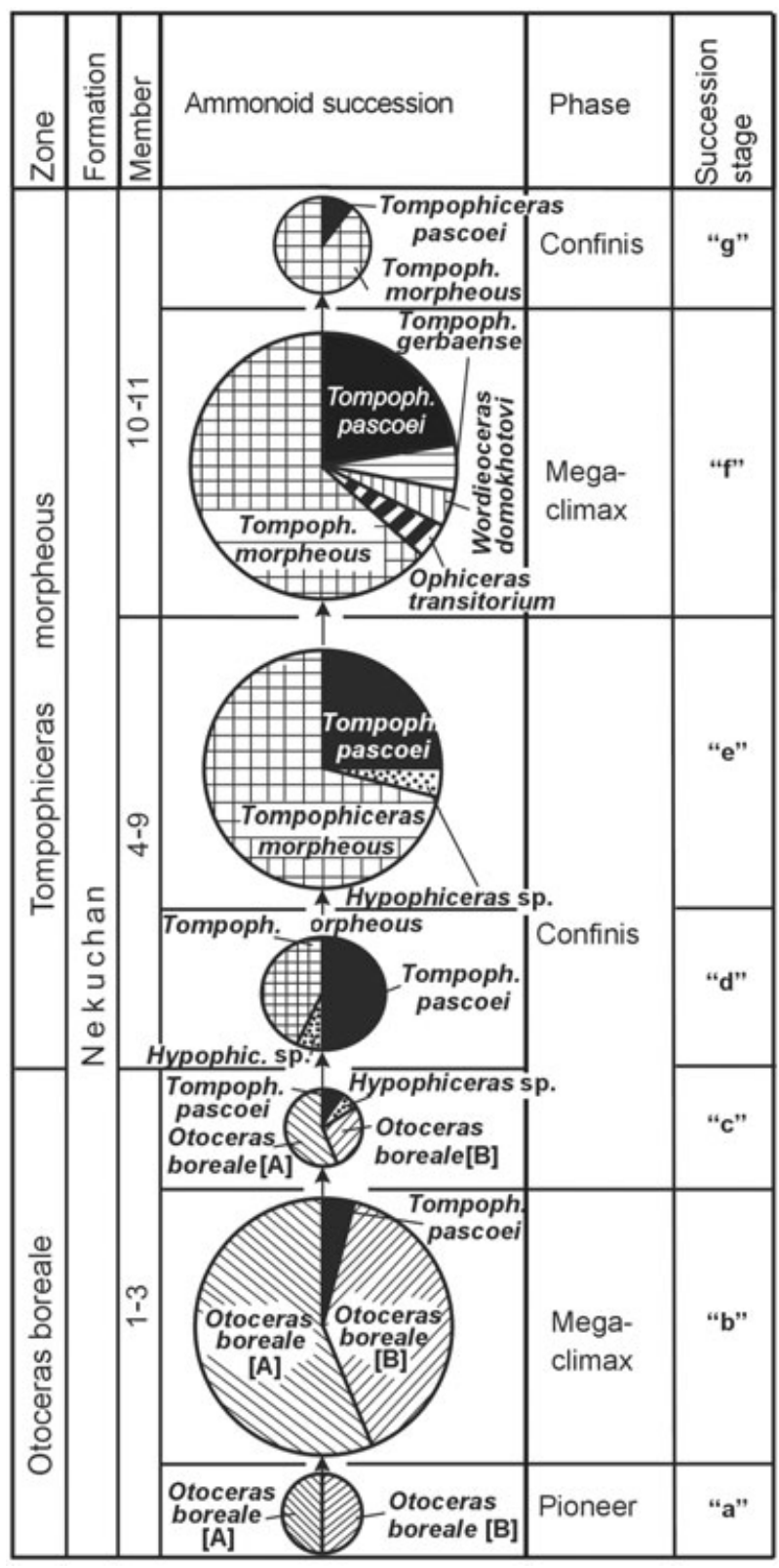

Fig. 3 Change of species composition and proportion in the early Induan ammonoid succession of the Setorym River section, Verkhoyansk area. Otoceras boreale (A) and Otoceras boreale (B) are different morphs of the same species, possibly sexual dimorphs. Observations were from 47 (stage a), 150 (stage b), 45 (stage c), 66 (stage d), 137 (stage e), 154 (stage f) and 56 (stage g) samples.

1971; Zakharov 2002). Changes of ammonoid dominance in the Setorym River section show that during the first three stages in development (stages a-c in Fig. 3), the most commonly occurring form in the assemblage is Otoceras boreale Spath, and during the following stages (d-g in Fig. 3) Tompophiceras morpheous Popow then became dominant. The population peak (megaclimax phase) of Otoceras occured in the development stage b represented in Fig. 3, which is characterized by some conodonts (e.g., Hindeodus typicalis [Sweet] and Clarkina cf. changhsingensis Wang and Wang; Kozur et al. 1995) and the first appearance of Tompophiceras (Tompophiceras pascoei [Spath]).

According to the traditional views (Griesbach 1880; Mojsisovics et al. 1895; Diener 1897; Kummel 1972; Tozer 1994), prior to definition at the Meishan Global Stratotype Section and Point (GSSP), the base Triassic in the Himalayas and other regions of the world is located at the base of the Otoceras beds. Using conodont data, Krystyn \& Orchard (1996), Kozur (1998) and Orchard \& Krystyn (1998) also placed the basal Triassic at the first occurrence of Otoceras in the Himalayas and Tibet. Basing their suggestion on their work with ophiceratid ammonoids, Krystyn \& Orchard (1996) were the first to suggest that Otoceras species of the Otoceras woodwardi Zone were younger than Boreal Otoceras. Kozur (1998) has also documented that the first very primitive Hindeodus parvus Kozur from Greenland are found above the Otoceras boreale Zone, in the Tompophiceras pascoei Zone. As the first appearance data (FAD) of H. parvus in the Himalayas and Tibet are at the base of the Otoceras woodwardi Zone, Kozur (1998) believes that the conodont data confirm Krystyn \& Orchard's (1996) idea concerning the younger age of the Otoceras woodwardi Zone compared with the Boreal Otoceras fauna. Conodont evidence with a bearing on the latest Permian age of the Otoceras boreale Zone has also been discussed (Wignall et al. 1996; Henderson \& Baud 1997; Beatty et al. 2006), but all of them need additional verification, above all because Permian-Triassic conodont assemblages from the Boreal realm are significantly less abundant and diversified compared with those from the Tethys, where the Meishan GSSP is located.

\section{Upper Olenekian-lowermost Anisian of the Olenek River area}

Many localities containing late Olenekian ammonoid faunas have been discovered in the Olenek River-Olenek Gulf area, but the most abundant and diversified ammonoid occurrences are found in the Mengilyakh Creek (Mojsisovics 1886; Lazurkin \& Korčinskaja 1963; Zaharov 1978; Zakharov 2007). The main known defect of the Lower Triassic Mengilyakh Creek section is its poor exposition.

Information on the upper Olenekian-lower Anisian sequence exposed along the Olenek River at the mouth of the Mengilyakh Creek is given in Table 1.

In the late Olenekian-earliest Anisian ammonoid succession of the Olenek River the highest diversity (megaclimax phase) occurs in the Olenikites spiniplicatus 
Table 1 The upper Olenekian-lower Anisian sequence exposed along the Olenek River at the mouth of the Mengilyakh Creek (72 $50^{\prime} 42.43^{\prime \prime} \mathrm{N}$, 12058'53.94"E; Mojsisovics 1886; Lazurkin \& Korčinskaja 1963; Zaharov 1978; Zakharov 2007), in descending order. The part of the Upper Olenekian exposed in this section is about $135 \mathrm{~m}$ thick.

\begin{tabular}{|c|c|c|c|c|c|}
\hline Formation/ zone & Member & Lithology & Thickness & Ammonoids & Other fossils \\
\hline \multirow[t]{3}{*}{$\begin{array}{l}\text { Lower Ulakhan-Krest } \\
\text { Formation/ Grambergia } \\
\text { taimyrensis Zone }\end{array}$} & 41 & $\begin{array}{l}\text { Black mudstone, intercalated } \\
\text { with black siltstone and grey, } \\
\text { fine-grained sandstone }\end{array}$ & $>20 \mathrm{~m}$ & & \\
\hline & 40 & $\begin{array}{l}\text { Black mudstone and siltstone } \\
\text { with thin layers }(1-20 \mathrm{~cm}) \text { of } \\
\text { dark grey, fine-grained } \\
\text { sandstone }\end{array}$ & $12 m$ & & \\
\hline & 39 & $\begin{array}{l}\text { Black mudstone and siltstone } \\
\text { with thin layers }(1-20 \mathrm{~cm}) \text { of } \\
\text { dark grey, fine-grained } \\
\text { sandstone }\end{array}$ & $7 \mathrm{~m}$ & $\begin{array}{l}\text { Prohungarites tuberculatus (Welter), } \\
\text { Prohungarites? sp. }\end{array}$ & \\
\hline \multirow[t]{2}{*}{$\begin{array}{l}\text { Pastanakhskaya and } \\
\text { Ystannakhskaya } \\
\text { formations/ Olenikites } \\
\text { spiniplicatus Zone }\end{array}$} & 38 & $\begin{array}{l}\text { Black mudstone and siltstone } \\
\text { with thin lenses of } \\
\text { fine-grained sandstone and } \\
\text { small, awkward-shaped } \\
\text { calcareous nodules }\end{array}$ & $3.2 \mathrm{~m}$ & $\begin{array}{l}\text { Prosphingites czekanowskii } \\
\text { Mojsisovics (dominant), } \\
\text { Nordophiceras euomphalus } \\
\text { (Keyserling), Subolenekites altus } \\
\text { (Mojsisovics), Olenekoceras } \\
\text { middendorffi (Keyserling), } \\
\text { Pseudosvalbardiceras sibiricum } \\
\text { (Mojsisovics) }\end{array}$ & \\
\hline & 37 & $\begin{array}{l}\text { Black mudstone with rare } \\
\text { large calcareous nodules (in } \\
\text { restricted and very low }\end{array}$ & ca. $14 \mathrm{~m}$ & & \\
\hline
\end{tabular}

outcrops)

36 Black mudstone with large calcareous nodules

$0.4 \mathrm{~m}$

Keyserlingites subrobustus

(Mojsisovics), Boreomeekoceras keyserlingi (Mojsisovics), Olenikites spiniplicatus (Mojsisovics), Nordophiceras popovi (Zakharov), Nordophiceras schmidti (Mojsisovics), Timoceras gracialis (Mojsisovics), Pseudosvalbardiceras sibiricum (Mojsisovics), Prosphingites czekanowskii Mojsisovics rare calcareous nodules

Black mudstone Black mudstone with small calcareous nodules outcrops) Black mudstone with large calcareous nodules Black mudstone with rare calcareous nodules Black mudstone with large calcareous nodules Black, striate mudstone with small calcareous nodules
$4.5 \mathrm{~m}$

$0.3 \mathrm{~m}$

$2.5 \mathrm{~m}$

$0.3 \mathrm{~m}$

$1.5 \mathrm{~m}$

$0.4 \mathrm{~m}$ Olenikites spiniplicatus (Mojsisovics) Olenikites spiniplicatus (Mojsisovics), Nordophiceras schmidti (Mojsisovics)

Olenikites spiniplicatus (Mojsisovics)

Olenikites spiniplicatus (Mojsisovics) (dominant), Nordophiceras schmidti (Mojsisovics)

$3.2 \mathrm{~m}$

$0.3 \mathrm{~m}$

Olenikites spiniplicatus (Mojsisovics)
Bivalves: Posidonia? sp., Mysidioptera sp.
Brachiopod: Lingula sp. Bivalve: Palaeoneilo sp. (dominant), Nordophiceras popovi (Zakharov), Boreomeekoceras keyserlingi (Mojsisovics), Olenekoceras middendorffi (Keyserling), Keyserlingites subrobustus (Mojsisovics)
Nautiloid:

Trematoceras cf. campanile (Mojsisovics) Belemnitid: Atractites sp. Nuculid bivalves 
Table 1 continued

\begin{tabular}{|c|c|c|c|c|c|}
\hline Formation/ zone & Member & Lithology & Thickness & Ammonoids & Other fossils \\
\hline & 27 & $\begin{array}{l}\text { Black mudstone with } \\
\text { numerous calcareous nodules }\end{array}$ & $9 \mathrm{~m}$ & $\begin{array}{l}\text { Olenekoceras middendorffi } \\
\text { (Keyserling) (dominant), Sibirites } \\
\text { eichwaldi Mojsisovics, Nordophiceras } \\
\text { schmidt (Mojsisovics), } \\
\text { Boreomeekoceras keyserlingi } \\
\text { (Mojsisovics) }\end{array}$ & $\begin{array}{l}\text { Plant: Pleuromeia } \\
\text { olenekensis Krassilov } \\
\text { Brachiopod: Lingula } \\
\text { sp. } \\
\text { Bivalve: Palaeoneilo } \\
\text { sp. } \\
\text { Scaphopods }\end{array}$ \\
\hline & 26 & $\begin{array}{l}\text { Black mudstone with rare } \\
\text { calcareous lenses and } \\
\text { nodules }\end{array}$ & $13 \mathrm{~m}$ & $\begin{array}{l}\text { Olenekoceras middendorffi } \\
\text { (Keyserling) (dominant), } \\
\text { Pseudosageceras boreale Zakharov, } \\
\text { Nordophiceras schmidti (Mojsisovics), } \\
\text { Boreomeekoceras keyserlingi } \\
\text { (Mojsisovics), Olenikites spiniplicatus } \\
\text { (Mojsisovics), Subolenekites altus } \\
\text { (Mojsisovics), Sibirites eichwaldi } \\
\text { Mojsisovics }\end{array}$ & \\
\hline & 25 & $\begin{array}{l}\text { Black mudstone with lenses of } \\
\text { limestone ("cone in cone" } \\
\text { structure) and rare calcareous } \\
\text { nodules }\end{array}$ & $20-25 \mathrm{~m}$ & & \\
\hline & 24 & $\begin{array}{l}\text { Black mudstone with small } \\
\text { calcareous nodules }\end{array}$ & $3 \mathrm{~m}$ & $\begin{array}{l}\text { Nordophiceras schmidti (Mojsisovics), } \\
\text { Olenikites spiniplicatus (Mojsisovics), } \\
\text { Subolenekites altus (Mojsisovics) }\end{array}$ & $\begin{array}{l}\text { Bivalves } \\
\text { Small gastropods }\end{array}$ \\
\hline & \multicolumn{5}{|c|}{ Closed interval, ca. 40-45 m } \\
\hline & 23 & $\begin{array}{l}\text { Black mudstone with small, } \\
\text { flat calcareous nodules }\end{array}$ & $18 \mathrm{~m}$ & $\begin{array}{l}\text { Nordophiceras euomphalus } \\
\text { (Keyserling), Olenikites spiniplicatus } \\
\text { (Mojsisovics), Subolenekites altus } \\
\text { (Mojsisovics), Olenekoceras } \\
\text { middendorffi (Keyserling) }\end{array}$ & \\
\hline & 22 & $\begin{array}{l}\text { Black mudstone with lenses of } \\
\text { limestone ("cone in cone" } \\
\text { structure) and small } \\
\text { calcareous nodules }\end{array}$ & $9 \mathrm{~m}$ & & \\
\hline & 21 & $\begin{array}{l}\text { Black mudstone with large } \\
\text { calcareous nodules }\end{array}$ & $1 \mathrm{~m}$ & $\begin{array}{l}\text { Nordophiceras schmidti (Mojsisovics), } \\
\text { Arctomeekoceras rotundatum } \\
\text { (Mojsisovics), Sibirites eichwaldi } \\
\text { Mojsisovics, Olenikites spiniplicatus } \\
\text { (Mojsisovics), Olenekoceras } \\
\text { middendorff (Keyserling) }\end{array}$ & $\begin{array}{l}\text { Brachiopod: Lingula } \\
\text { sp } \\
\text { Bivalve: Posidonia? sp. }\end{array}$ \\
\hline & 20 & $\begin{array}{l}\text { Black mudstone with lenses of } \\
\text { limestone ("cone in cone" } \\
\text { structure) and large } \\
\text { calcareous nodules }\end{array}$ & $16 \mathrm{~m}$ & & \\
\hline & 19 & $\begin{array}{l}\text { Greenish grey mudstone and } \\
\text { siltstone with rare calcareous } \\
\text { nodules }\end{array}$ & $0.2 \mathrm{~m}$ & $\begin{array}{l}\text { Prosphingites czekanowskii } \\
\text { Mojsisovics }\end{array}$ & \\
\hline & 18 & $\begin{array}{l}\text { Black mudstone with lenses of } \\
\text { limestone ("cone in cone" } \\
\text { structure), sandy limestone } \\
\text { and rare calcareous nodules }\end{array}$ & $4 \mathrm{~m}$ & & \\
\hline & 17 & $\begin{array}{l}\text { Black mudstone with } \\
\text { calcareous nodules }\end{array}$ & $0.3 \mathrm{~m}$ & $\begin{array}{l}\text { Nordophiceras schmidti (Mojsisovics), } \\
\text { Pseudosvalbardiceras sibiricum } \\
\text { (Mojsisovics) }\end{array}$ & $\begin{array}{l}\text { Nautiloid: } \\
\text { Phaedrysmocheilus } \\
\text { olenekensis (Zakharov) }\end{array}$ \\
\hline & 16 & Black mudstone & $1 \mathrm{~m}$ & & \\
\hline & 15 & $\begin{array}{l}\text { Black mudstone with } \\
\text { numerous calcareous nodules }\end{array}$ & $0.3 \mathrm{~m}$ & $\begin{array}{l}\text { Olenikites spiniplicatus (Mojsisovics), } \\
\text { Olenekoceras middendorffi } \\
\text { (Keyserling) }\end{array}$ & \\
\hline
\end{tabular}


Table 1 continued

\begin{tabular}{|c|c|c|c|c|c|}
\hline Formation/ zone & Member & Lithology & Thickness & Ammonoids & Other fossils \\
\hline & 14 & Black mudstone & $1 \mathrm{~m}$ & & \\
\hline & 13 & $\begin{array}{l}\text { Black mudstone with } \\
\text { numerous large calcareous } \\
\text { nodules }\end{array}$ & $0.5 \mathrm{~m}$ & $\begin{array}{l}\text { Nordophiceras schmidti (Mojsisovics), } \\
\text { Nordophiceras euomphalus } \\
\text { (Keyserling), Olenikites spiniplicatus } \\
\text { (Mojsisovics), Timoceras gracialis } \\
\text { (Mojsisovics), Olenekoceras } \\
\text { middendorffi (Keyserling), } \\
\text { Keyserlingites subrobustus } \\
\text { (Mojsisovics) }\end{array}$ & $\begin{array}{l}\text { Bivalve: Mysidioptera } \\
\text { aurita Popow } \\
\text { Nautiloids: } \\
\text { Trematoceras cf. } \\
\text { campanile } \\
\text { (Mojsisovics), } \\
\text { Phaedrysmocheilus } \\
\text { olenekensis (Zakharov) }\end{array}$ \\
\hline & 12 & Black mudstone & $0.7 \mathrm{~m}$ & & \\
\hline & 11 & $\begin{array}{l}\text { Black mudstone with lenses of } \\
\text { limestone ("cone in cone" } \\
\text { structure) and large } \\
\text { calcareous nodules }\end{array}$ & $0.3 \mathrm{~m}$ & $\begin{array}{l}\text { Nordophiceras schmidti (Mojsisovics) } \\
\text { (dominant), Pseudosvalbardiceras } \\
\text { sibiricum (Mojsisovics), Olenikites } \\
\text { spiniplicatus (Mojsisovics), } \\
\text { Olenekoceras middendorffi } \\
\text { (Keyserling) }\end{array}$ & \\
\hline & 9 & $\begin{array}{l}\text { Black mudstone with lenses of } \\
\text { limestone ("cone in cone" } \\
\text { structure) and calcareous } \\
\text { nodules }\end{array}$ & $0.5 \mathrm{~m}$ & $\begin{array}{l}\text { Nordophiceras schmidti (Mojsisovics), } \\
\text { Olenikites spiniplicatus (Mojsisovics), } \\
\text { Timoceras gracialis (Mojsisovics) }\end{array}$ & $\begin{array}{l}\text { Nautiloid: } \\
\text { Phaedrysmocheilus } \\
\text { olenekensis (Zakharov) }\end{array}$ \\
\hline & 8 & $\begin{array}{l}\text { Black mudstone with rare } \\
\text { calcareous nodules }\end{array}$ & $3 \mathrm{~m}$ & $\begin{array}{l}\text { Nordophiceras schmidti (Mojsisovics), } \\
\text { Olenikites spiniplicatus (Mojsisovics), } \\
\text { Timoceras gracialis (Mojsisovics) }\end{array}$ & $\begin{array}{l}\text { Bivalves: Posidonia } \\
\text { sp., Mysidioptera sp. } \\
\text { Nautiloids: } \\
\text { Trematoceras sp., } \\
\text { Phaedrysmocheilus } \\
\text { olenekensis (Zakharov) } \\
\text { Gastropods }\end{array}$ \\
\hline & 7 & $\begin{array}{l}\text { Black mudstone with } \\
\text { numerous large calcareous } \\
\text { nodules }\end{array}$ & $0.5 \mathrm{~m}$ & $\begin{array}{l}\text { Olenekoceras middendorffi } \\
\text { (Keyserling) (dominant), } \\
\text { Pseudosageceras boreale Zakharov, } \\
\text { Boreomeekoceras keyserlingi } \\
\text { (Mojsisovics), Nordophiceras popovi } \\
\text { (Zakharov), Arctomeekoceras } \\
\text { rotundatum (Mojsisovics), Sibirites } \\
\text { eichwaldi Mojsisovics, Subolenekites } \\
\text { altus (Mojsisovics) }\end{array}$ & $\begin{array}{l}\text { Belemnite: Atractites } \\
\text { aff. boecki } \\
\text { (Stürzenbaum) } \\
\text { Gastropods }\end{array}$ \\
\hline & 6 & $\begin{array}{l}\text { Black mudstone with very } \\
\text { rare calcareous nodules }\end{array}$ & $6 \mathrm{~m}$ & & \\
\hline & 5 & $\begin{array}{l}\text { Black mudstone with } \\
\text { numerous calcareous nodules }\end{array}$ & $1 \mathrm{~m}$ & $\begin{array}{l}\text { Olenekoceras middendorffi } \\
\text { (Keyserling) (dominant), } \\
\text { Boreomeekoceras keyserlingi } \\
\text { (Mojsisovics), Sibirites eichwaldi } \\
\text { Mojsisovics, Olenikites spiniplicatus } \\
\text { (Mojsisovics), Subolenekites altus } \\
\text { (Mojsisovics) }\end{array}$ & \\
\hline & 4 & Black mudstone & $1.5 \mathrm{~m}$ & & \\
\hline & 3 & $\begin{array}{l}\text { Black mudstone with } \\
\text { numerous large calcareous } \\
\text { nodules }\end{array}$ & $0.5 \mathrm{~m}$ & $\begin{array}{l}\text { Olenekoceras middendorffi (Keyserlig) } \\
\text { (dominant), Nordophiceras } \\
\text { euomphalus (Keyserling), } \\
\text { Arctomeekoceras sp., } \\
\text { Boreomeekoceras keyserlingi } \\
\text { (Mojsisovics), Subolenekites altus } \\
\text { (Mojsisovics), Sibirites eichwaldi } \\
\text { Mojsisovics }\end{array}$ & $\begin{array}{l}\text { Bivalves } \\
\text { Gastropods }\end{array}$ \\
\hline
\end{tabular}


Table 1 continued

\begin{tabular}{|c|c|c|c|c|c|}
\hline Formation/ zone & Member & Lithology & Thickness & Ammonoids & Other fossils \\
\hline & 2 & $\begin{array}{l}\text { Black mudstone with rare } \\
\text { small calcareous nodules }\end{array}$ & $12 \mathrm{~m}$ & $\begin{array}{l}\text { Nordophiceras euomphalus } \\
\text { (Keyserling), Arctomeekoceras } \\
\text { rotundatum (Mojsisovics), } \\
\text { Subolenekites altus (Mojsisovics), } \\
\text { Boreomeekoceras keyserlingi } \\
\text { (Mojsisovics), Keyserlingites } \\
\text { subrobustus (Mojsisovics) }\end{array}$ & \\
\hline $\begin{array}{l}\text { Parasibirites } \\
\text { grambergi Zone } \\
\text { (upper part) }\end{array}$ & 1 & $\begin{array}{l}\text { Black mudstone with small } \\
\text { calcareous nodules (in } \\
\text { restricted outcrops) }\end{array}$ & $30 \mathrm{~m}$ & $\begin{array}{l}\text { Parasibirites grambergi Popow, } \\
\text { Sibirites pretiosus Mojsisovics. In } \\
\text { removed nodules near outcrops: } \\
\text { Pseudosageceras, Nordophiceras, } \\
\text { Boreomeekoceras, Olenikites, } \\
\text { Subolenekites, Sibirites, Olenekoceras }\end{array}$ & \\
\hline
\end{tabular}

Zone, where Olenikites spiniplicatus (Mojsisovics) dominates the ammonoid assemblage (Zakharov 2007: fig. 3). Representatives of the genus Olenekoceras are more frequent in this succession than are Keyserlingites. In Arctic Siberia Olenekoceras are found within two late Olenekian zones, the Parasibirites grambergi and Olenikites spiniplicatus zones (Dagys \& Ermakova 1988), which apparently correspond with the Olenekoceras-bearing Neocolumbites insignis-Subfengshanites multiformis interval in the middle-latitude south Russian Far East area (Zakharov 1997).

\section{Upper Olenekian-Anisian of South Primorye (Russian Island and Atlasov Cape area)}

In the late Olenekian-earliest Anisian ammonoid succession the megaclimax phase corresponds with the Neocolumbites insignis Zone (Zakharov 1997; Zakharov, Popov et al. 2005). This phase is characterized by the presence of some Boreal elements (e.g., Olenekoceras and Nordophiceras; Fig. 4). A marked increase in the abundance of Columbites, Subfengshanites and Ussuriphyllites took place during the stages a-c depicted in Fig. 4.

\section{Upper Olenekian of Mangyshlak (Dolnapa)}

Information on the middle Olenekian-lower Anisian sequence exposed near the Dolnapa (Angry Sister) drawwell is given in Table 2 .

During the early part of the Late Olenekian megaclimax (the Columbites parisianus-Procolumbites karatauchicus interval), "Dinarites" asiaticus Shevyrev, associated with diverse articulate brachiopods (Piarorhynchella, Lissorhynchia, Prolissorhynchia, Sinuplicorhynchia, Hustedtiella, Spirigerellina, Lepismatina, Antezeilleria, Thyratryaria, Proanadyrella and others) and abundant foraminifera, dominated the ammonoid assemblage (Zakharov \& Popov 2007; fig. 3). This dominance was replaced by Stacheites undatus (Astachova) by the end of the Olenekian. The latter species is associated with representatives of the genus Arnautoceltites, usually a latest Olenekian element in the Tethyan assemblages. The main peculiarity of Olenekian successions is the development of the Tirolites fauna. Tirolites, the typical tropical element in the Tethys, arose during the middle Olenekian climatic optimum, reaching a maximal population at that time. However, it continued to occur during the late Olenekian in Mangyshlak, as well as in many other low-latitude regions (Idaho, Caucasus, Alps, Iran and Albania), and at middle latitudes (South Primorye).

\section{Discussion}

Palynological, petrographical and isotopic records show that the global warming following the PermoCarboniferous glaciation was probably caused primarily

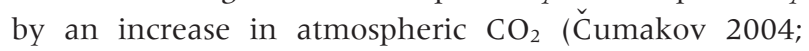
Korte, Jasper et al. 2005; Angiolini et al. 2006). New palaeobiogeographical evidence (Figs. 5-9) suggests marked climatic changes also occurred through the post-Sakmarian Permian, and Early and Middle Triassic, when frequent expansions and reductions of the warmtemperate climatic zones in the high and middle latitudes of the Northern and Southern hemispheres apparently took place. This is consistent with some known geochemical patterns, discussed below. However, temperatures estimated from oxygen isotopic analyses on Permian and Lower-Middle Triassic biogenic carbonates (mainly brachiopod calcite), are very restricted because of the lack of well-preserved material for isotope investigation. Our recent data (Zaharov et al. 2008) suggest a warming maximum existed in the Late Kungurian, with 


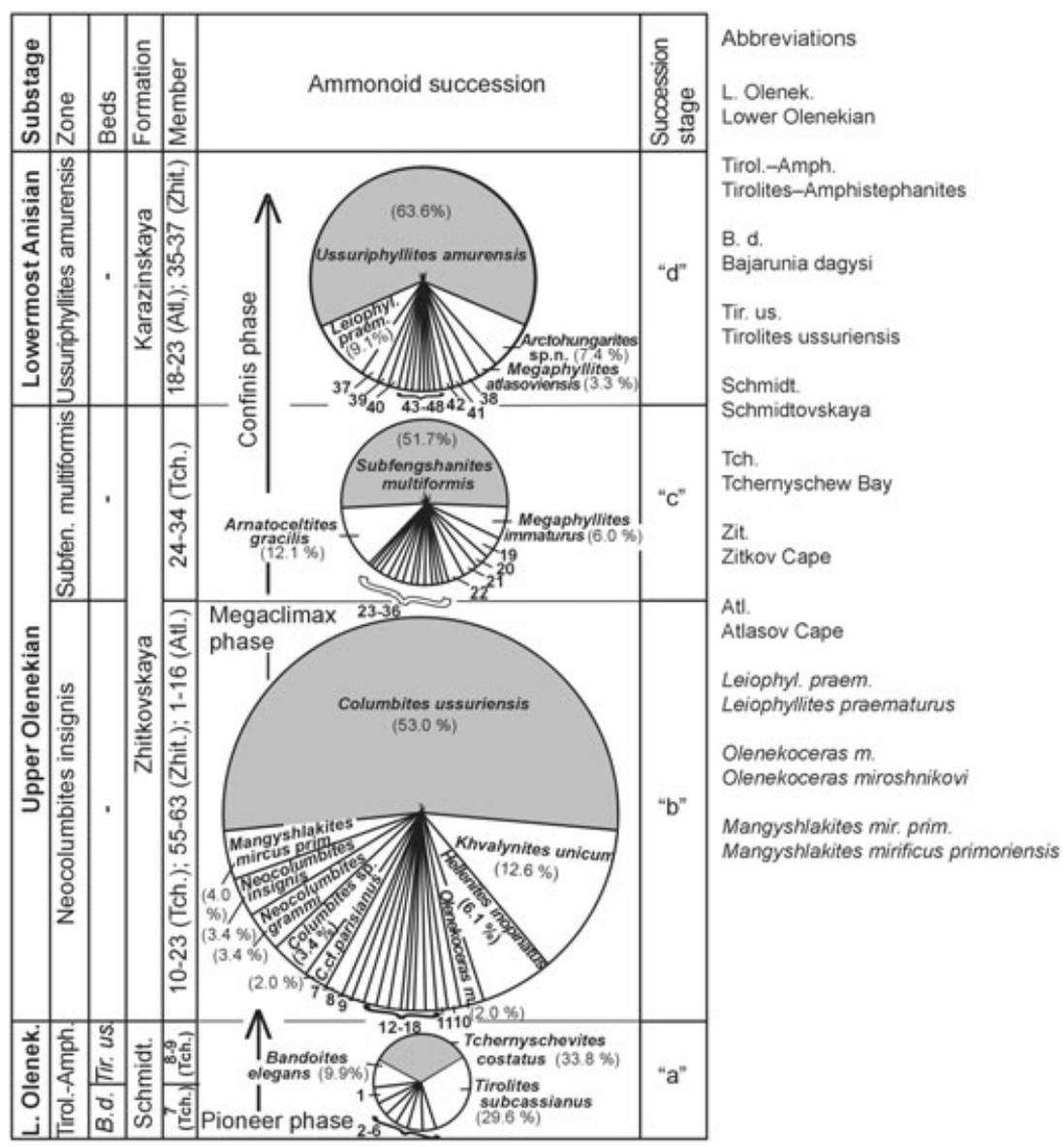

Fig. 4 Change of species composition and proportion in the Olenekian-earliest Anisian ammonoid succession of Russian Island-Atlasov Cape area, South Primorye. 1, Tirolites ussuriensis Zharnikova; 2-6, others, including Amphistephanites parisensis (Zakharov), Kazakhstanites sonticus (Zakharov), Tchernyschevites subdalmatus Zakharov, Bajarunia dagysi Zakharov and Kazakhstanites zakharovi Zharnikova; 7, Olenekoceras tebenkovi (Zharnikova); 8, Procolumbites subquadratus Burij \& Zharnikova; 9, Procolumbites sp.; 10, Hellenites tchernyschewiensis Zakharov; 11, Olenekoceras meridianus (Zakharov); 12-18, others, including Olenekoceras miroshnikovi Burij \& Zharnikova, "Dieneroceras" spathi Kummel \& Steele, Svalbardiceras sp., Svalbardiceras zhitkovense Zakharov, Preflorianites venustus Zakharov, Tirolites cf. subcassianus Zakharov, Subdoricranites? sp.; 19, Zhitkovites insularis (Kiparisova); 20, Pseudoprosphingites globosus (Kiparisova); 21, Isculitoides? suboviformis (Kiparisova); 22, Sulioticeras maritimus (Kiparisova); 23-36, others, including Columbites sp., Prenkites aff. timorensis Spath, Palaeophyllites superior Zakharov, Arnautoceltites sp., Pseudosageceras longilobatum Kiparisova, Pseudosageceras simplex Kiparisova, Pseudoprosphingites aff. globosus (Kiparisova), Isculitoides? aff. suboviformis (Kiparisova), Leiophyllites praematurus Kiparisova, Danubites aff. floriani Mojsisovics, Danubites admaris Kiparisova, Danubites insertus Kiparisova, "Dieneroceras" karazini (Kummel \& Teichert); 36, Prohungarites popowi; 37, Arctohungarites primoriensis; 38, Paracrochordiceras sp. nov.; 39, Paradanubites sp.; 40, Leiophyllites sp.; 41, Tropigastrites sublahontanum; 42-48, others, including Prohungarites? sp., Parasageceras sp. nov., Salterites sp., Ussurites sp., Arctohungarites sp., Keyserlingites? sp., Prionitidae gen. et sp. nov. Observations were from 75 (stage a), 300 (stage b), 130 (stage c) and 170 (stage d) samples.

palaeotemperatures in Spitsbergen of not lower than $23.1-23.8^{\circ} \mathrm{C}$, and apparently also suggest short-term cooling in the latest part of the early Capitanian (Zaharov et al. 2008), as well as in the latest Wordian (Kotlyar et al. 2006; Shi 2006). This is because a sharp drop in palaeotemperatures (from 20.4 to $16.5^{\circ} \mathrm{C}$; Zaharov et al. 2008) had occurred in successions of the earliest part of the late Wordian-early Capitanian interval at high latitudes of the Far East (Russkaya-Omolonskaya and Khivach areas of the Omolon River basin). Naturally, the early Capitanian temperature drop, so far documented only in the Omolon River basin, could only be localized.

Two maxima in palaeowater temperatures seem to have occurred during the Late Permian. One was during the early Wuchiapingian, with palaeotemperatures of $25.2-27.9^{\circ} \mathrm{C}$ calculated for the middle palaeolatitudes of Transcaucasia (Zaharov et al. 2001). The other maximum occurred during the late Changhsingian, at both the middle and high palaeolatitudes, and was characterized by somewhat lower palaeotemperatures: $22.0-24.2^{\circ} \mathrm{C}$ for 


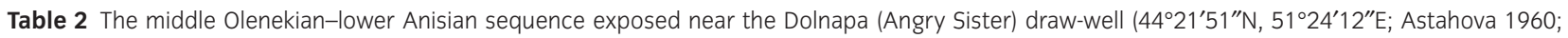
Ševyrev 1968, 2002; Gavrilova 1980, 1989; Balini et al. 2000; Zakharov \& Popov 2007), in descending order.

\begin{tabular}{|c|c|c|c|c|c|}
\hline $\begin{array}{l}\text { Formation/ } \\
\mathrm{bed} / \text { zone }\end{array}$ & Member & Lithology & Thickness & Ammonoids & Other fossils \\
\hline \multirow{10}{*}{$\begin{array}{l}\text { Lower } \\
\text { Karaduanskaya } \\
\text { Formation } \\
\text { (Lower Anisian) }\end{array}$} & 32 & $\begin{array}{l}\text { Intercalation of grey, } \\
\text { fine-grained sandstone and } \\
\text { greenish grey mudstone }\end{array}$ & $>130 \mathrm{~m}$ & & \\
\hline & 31 & $\begin{array}{l}\text { Black and greenish grey } \\
\text { mudstone }\end{array}$ & ca. $40 \mathrm{~m}$ & & \\
\hline & 30 & $\begin{array}{l}\text { Intercalation of black } \\
\text { mudstone and grey, } \\
\text { fine-grained sandstone }\end{array}$ & ca. $100 \mathrm{~m}$ & & \\
\hline & 29 & $\begin{array}{l}\text { Intercalation of grey, } \\
\text { intermediate-grained and } \\
\text { fine-grained sandstone }\end{array}$ & $5 \mathrm{~m}$ & & \\
\hline & 28 & Grey, fine-grained sandstone & $10 \mathrm{~m}$ & & \\
\hline & 27 & $\begin{array}{l}\text { Black siltstone with thin } \\
\text { interlayers of siliceous rocks }\end{array}$ & $17 \mathrm{~m}$ & & \\
\hline & 26 & $\begin{array}{l}\text { Grey, intermediate-grained } \\
\text { and fine-grained sandstone }\end{array}$ & ca. $110 \mathrm{~m}$ & & \\
\hline & 25 & $\begin{array}{l}\text { Intercalation of greenish grey, } \\
\text { fine-grained sandstone and } \\
\text { mudstone with interlayers } \\
\text { of limestone-coquina } \\
\text { (bivalve molluscs) }\end{array}$ & $17 \mathrm{~m}$ & & \\
\hline & 24 & $\begin{array}{l}\text { Brown, intermediate-grained } \\
\text { sandstone }\end{array}$ & $30 \mathrm{~m}$ & & \\
\hline & 23 & $\begin{array}{l}\text { Brown and grey intermediate- } \\
\text { and fine-grained sandstone }\end{array}$ & $19 m$ & & \\
\hline \multirow{5}{*}{$\begin{array}{l}\text { Lower } \\
\text { Karadzhatykskaya } \\
\text { Formation } \\
\text { (total thickness } \\
\text { ca. } 324 \text { m; } \\
\text { Upper } \\
\text { Olenekian)/ } \\
\text { Eumorphotis } \\
\text { beds }\end{array}$} & 22 & $\begin{array}{l}\text { Intercalation of greenish grey } \\
\text { siltstone and grey, } \\
\text { fine-grained, calcareous } \\
\text { sandstone }\end{array}$ & $34 \mathrm{~m}$ & & $\begin{array}{l}\text { Bivalves: Pteria sp., } \\
\text { Leptochondria sp. (specimens } \\
409-11 \text { and } 410-1 \text { collected from } \\
\text { different beds) }\end{array}$ \\
\hline & 21 & Grey, fine-grained sandstone & $7.5 \mathrm{~m}$ & & \\
\hline & 20 & $\begin{array}{l}\text { Grey, fine-grained sandstone } \\
\text { with interlayers of mudstone } \\
\text { and rare lenses of limestone }\end{array}$ & ca. 75 m & $\begin{array}{l}\text { Pseudosageceras sp. (specimen } \\
\text { 409-10) }\end{array}$ & $\begin{array}{l}\text { Bivalve: Eumorphotis sp. } \\
\text { (specimen 409-13) } \\
\text { Nautiloid: Trematoceras sp. }\end{array}$ \\
\hline & 19 & $\begin{array}{l}\text { Greenish-grey mudstone with } \\
\text { rare interlayers }(20-30 \mathrm{~cm}) \text { of } \\
\text { grey, fine-grained sandstone } \\
\text { and lenses of limestone }\end{array}$ & $27 \mathrm{~m}$ & $\begin{array}{l}\text { Badly preserved specimens (409-9 } \\
\text { and } 409-10, \text { collected from different } \\
\text { beds) }\end{array}$ & $\begin{array}{l}\text { Bivalve: Pteria sp. } \\
\text { Gastropods } \\
\text { Nautiloids (Nautilida) }\end{array}$ \\
\hline & 18 & $\begin{array}{l}\text { Grey, fine-grained sandstone } \\
\text { with interlayers of greenish } \\
\text { grey mudstone }\end{array}$ & $8 \mathrm{~m}$ & & \\
\hline $\begin{array}{l}\text { Arnautoceltites } \\
\text { bajarunasi- } \\
\text { Stacheites } \\
\text { undatus Zone }\end{array}$ & 17 & $\begin{array}{l}\text { Intercalation of greenish grey } \\
\text { mudstone with calcareous } \\
\text { boulders, siltstone with plant } \\
\text { detritus and fine-grained } \\
\text { sandstone with interlayers of } \\
\text { light-grey limestone }\end{array}$ & ca. $165 \mathrm{~m}$ & $\begin{array}{l}\text { From the upper part: Kashmiritidae } \\
\text { gen. et sp. indet., Albanites gracilis } \\
\text { (Kiparisova), Preflorianites sp., } \\
\text { Stacheites concavus Shevyrev, } \\
\text { Stacheites undatus (Astachova), } \\
\text { "Dinarites" orientalis Shevyrev, } \\
\text { Tirolites armatus Shevyrev (409-1, } \\
-4,-5,-6 ; 408-5,-6,-7 \text { collected } \\
\text { from several beds). } \\
\text { From the lower part: } \\
\text { Pseudosageceras sp., Kazakhstanites } \\
\text { dolnapaensis Shevyrev, Albanites } \\
\text { gracilis (Kiparisova), "Dinarites" sp., } \\
\text { Stacheites concavus Shevyrev, } \\
\text { Stacheites undatus (Astachova), }\end{array}$ & $\begin{array}{l}\text { From the upper part: crinoids; } \\
\text { brachiopods, Piarorhynchella } \\
\text { mangyshlakensis Dagys, } \\
\text { Lissorhynchia sp. nov., } \\
\text { Sinuplicorhynchia? sp. nov., } \\
\text { Thyratryaria aff. pertumida Xu } \\
\text { and Liu, Lepismatina sp. nov., } \\
\text { Spirigerellinae gen. et sp. indet. } \\
\text { (409-3, -4, -7 collected from } \\
\text { different beds); bivalves, } \\
\text { Palaeoneilo sp., Neoschisodus sp. } \\
\text { (408-5); nautiloid, Trematoceras } \\
\text { sp. (409-1). } \\
\text { From the lower part: bivalves, } \\
\text { Leda sp., Palaeoneillo sp., }\end{array}$ \\
\hline
\end{tabular}


Table 2 continued

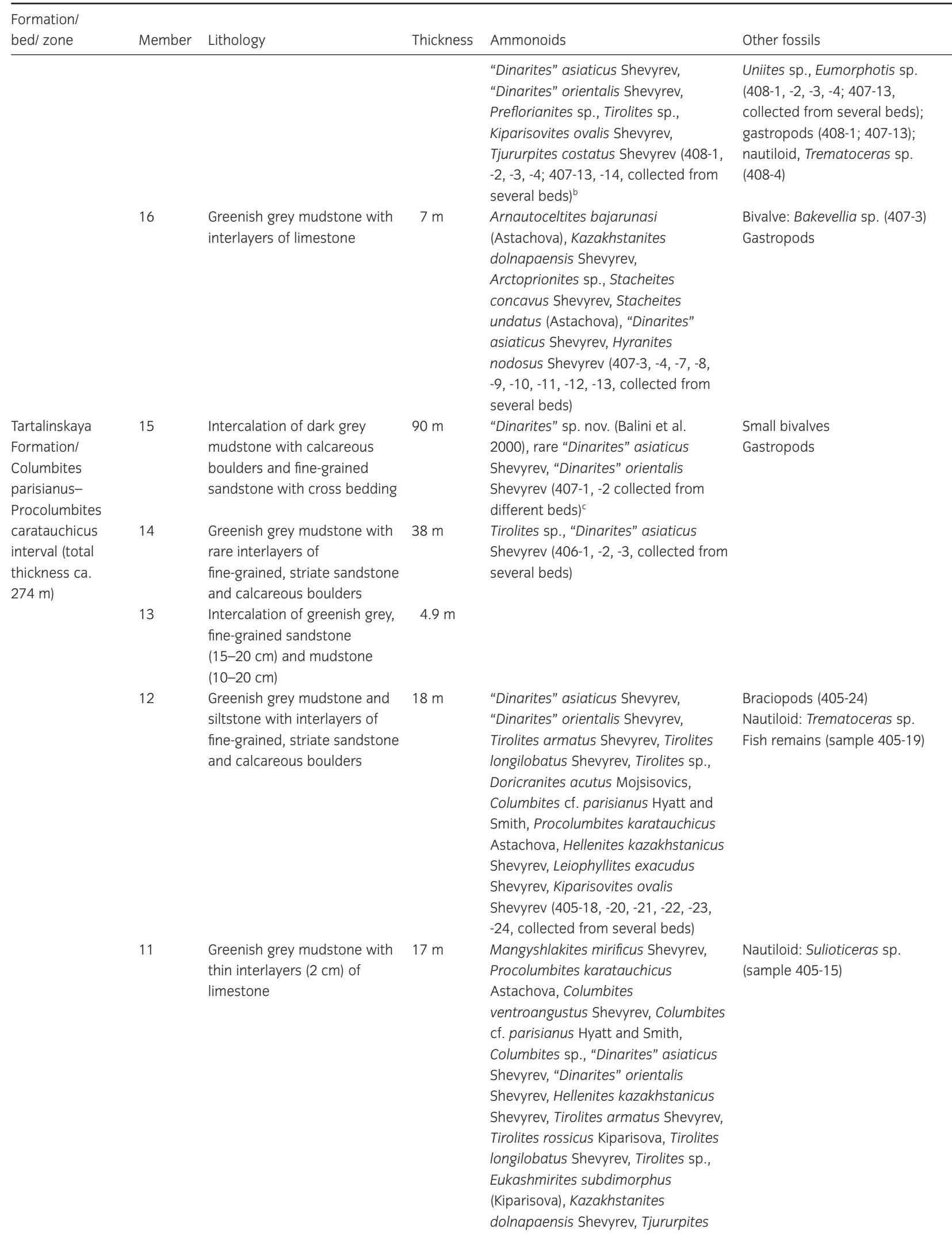


Table 2 continued

\begin{tabular}{|c|c|c|c|c|c|}
\hline $\begin{array}{l}\text { Formation/ } \\
\text { bed/zone }\end{array}$ & Member & Lithology & Thickness & Ammonoids & Other fossils \\
\hline & 10 & $\begin{array}{l}\text { Grey, fine-grained sandstone } \\
\text { and greenish grey mudstone } \\
\text { with rare lenses of limestone }\end{array}$ & $6 \mathrm{~m}$ & $\begin{array}{l}\text { costatus Shevyrev (405-14, }-15,-16, \\
-17,-18, \text { collected from several beds) } \\
\text { Tirolites sp., Hellenites } \\
\text { kazakhstanicus Shevyrev (405-13) }\end{array}$ & \\
\hline & 9 & $\begin{array}{l}\text { Greenish grey mudstone and } \\
\text { siltstone with interlayers } \\
(5-15 \mathrm{~cm}) \text { of fine-grained, } \\
\text { striate sandstone, calcareous } \\
\text { lenses and boulders }\end{array}$ & ca. $100 \mathrm{~m}$ & $\begin{array}{l}\text { From the upper part: Doricranites } \\
\text { bogdoanus (Buch), Prionitidae gen. et } \\
\text { sp. indet., Tirolites armatus Shevyrev, } \\
\text { Tirolites sp., "Dinarites" asiaticus } \\
\text { Shevyrev, "Dinarites" orientalis } \\
\text { Shevyrev, Columbites ventroangustus } \\
\text { Shevyrev, Columbites cf. parisianus } \\
\text { Hyatt and Smith, Columbites sp., } \\
\text { Procolumbites karatauchicus } \\
\text { Astachova, Hellenites kazakhstanicus } \\
\text { Shevyrev, Hellenites sp., Leiophyllites } \\
\text { exacudus Shevyrev (405-9, -10, -11, } \\
-12,-12 a,-18,-22,-23, \text { collected from } \\
\text { several beds). } \\
\text { From the lower part: } \\
\text { Pseudosageceras longilobatum } \\
\text { Kiparisova, Doricranites acutus } \\
\text { (Moisisovics), Prionitidae gen. et sp. } \\
\text { indet., "Dinarites" asiaticus Shevyrev, } \\
\text { "Dinarites" orientalis Shevyrev, } \\
\text { Tirolites sp., Albanites gracilis } \\
\text { (Kiparisova), Columbites sp., } \\
\text { Preflorianites kiparisovae Shevyrev, } \\
\text { Leiophyllites exacudus Shevyrev } \\
\text { (405-1, 404-22, -24, -25, -26, -28, -36, } \\
-41,-48,-55,-57,-59,-60,-61,-65, \\
405-2,-7, \text { collected from several } \\
\text { beds) }\end{array}$ & $\begin{array}{l}\text { From the upper part: crinoids } \\
\text { (405-10); brachiopods, } \\
\text { Piarorhynchella } \\
\text { mangyshlakensis Dagys, } \\
\text { Lissorhynchia sp. nov., } \\
\text { Hustedtiella planicosta Dagys, } \\
\text { Lepismatina sp. nov., } \\
\text { Prelissorhynchia sp. nov., } \\
\text { Antezeilleria sp. (405-9); } \\
\text { bivalves, Bakevellia sp., } \\
\text { Leptochondria cf. minima } \\
\text { (Kiparisova), Eumorphotis sp., } \\
\text { Palaeoneilo sp., Mytilidae gen. } \\
\text { et sp. indet. (405-9, -10, -11, } \\
\text { collected from several beds); } \\
\text { gastropods (405-10, -14); } \\
\text { nautiloids, Phaedrysmocheilus } \\
\text { sp., Nautilidae gen. et sp. } \\
\text { indet., Trematoceras sp. } \\
\text { (405-10, -11, -12 collected from } \\
\text { several beds). } \\
\text { From the lower part: crinoids } \\
\text { (404-61); brachiopods, Lingula } \\
\text { sp., Piarorhynchella } \\
\text { mangyshlakensis Dagys, } \\
\text { Spirigerellina sp., Lissorhynchia } \\
\text { sp. nov., Spirigerellina pygmaea } \\
\text { Dagys, Lepismatina sp. nov., } \\
\text { Thyratryaria sp. nov. A, } \\
\text { Thyratryaria sp. nov. B, } \\
\text { Proanadyrella? sp., } \\
\text { Spirigerellinae gen. et sp. indet. } \\
\text { (405-2, -4, -7; } 404-47,-49,-50, \\
\text {-59, -61, collected from several } \\
\text { beds); bivalves, Eumorphotis } \\
\text { sp., Entolium sp. (404-19, -35, } \\
\text {-59, -61, collected from several } \\
\text { beds); gastropods, (404-59, -61 } \\
\text { collected from different beds); } \\
\text { nautiloids, Phaedrysmocheilus } \\
\text { sp., Trematoceras sp. (405-4, } \\
\text { 404-39, collected rom different } \\
\text { beds) }\end{array}$ \\
\hline $\begin{array}{l}\text { Kiparisovites } \\
\text { carinatus- } \\
\text { Tirolites } \\
\text { cassianus } \\
\text { Zone } \\
(103)^{\mathrm{a}}\end{array}$ & 8 & $\begin{array}{l}\text { Greenish grey siltstone with } \\
\text { interlayers }(10 \mathrm{~cm}) \text { of } \\
\text { fine-grained sandstone } \\
\text { calcareous boulders }\end{array}$ & $17 \mathrm{~m}$ & $\begin{array}{l}\text { Tirolites armatus Shevyrev, Albanites } \\
\text { gracilis (Kiparisova), Kazakhstanites } \\
\text { dolnapaensis Shevyrev, Tjururpites } \\
\text { cf. costatus Shevyrev, "Dinarites" } \\
\text { orientalis Shevyrev (404-1, -7, -9, -15, } \\
\text { collected from several beds) }\end{array}$ & $\begin{array}{l}\text { Sea-urchins (spines) (404-5); } \\
\text { crinoids (404-5); brachiopods, } \\
\text { Lepismatina sp. nov., } \\
\text { Piarorhynchella } \\
\text { mangyshlakensis Dagys (404-1, } \\
-9,-13 \text {, collected from several }\end{array}$ \\
\hline
\end{tabular}

Kiparisovites carinatuscassianus $(103 m)^{a}$ 
Table 2 continued

Formation/ $\mathrm{bed/zone}$ Member Lithology

Thickness

Ammonoids

Other fossils

beds); small gastropods (404-9); nautiloids, Trematoceras sp., Phaedrysmocheilus sp. (404-6, -9 , collected from different beds). Upper part of the Tirolites beds contains foraminiferal remains (Vuks 1997)

7 Greenish grey siltstone with

$11 \mathrm{~m}$ calcareous boulders and lenses $(15 \mathrm{~cm})$ of limestone

6 Intercalation of greenish grey siltstone $(50 \mathrm{~cm})$, fine-grained, calcareous, wavy and cross-bedding sandstone $(5-10 \mathrm{~cm})$

5 Greenish grey siltstone with thin interlayers $(2-5 \mathrm{~cm})$ of brown limestone

4 Greenish grey mudstone with thin interlayers $(5-6 \mathrm{~cm})$ of fine-grained sandstone and calcareous boulders

\section{3}

Intercalation of greenish grey

$2 \mathrm{~m}$ siltstone $(0.5 \mathrm{~m})$ and fine-grained sandstone Greenish grey siltstone with $4 \mathrm{~m}$ calcareous boulders

1

$3 m$

$40 \mathrm{~m}$
Tirolites sp., "Dinarites" asiaticus Shevyrev, "Dinarites" orientalis Shevyrev (404-1, -2, -3, collected from several beds)

Kiparisovites carinatus Astachova, "Dinarites" orientalis Shevyrev (403-37, -38, -38a, collected from several beds)

ca. $30 \mathrm{~m}$ From the upper part: Pseudosageceras sp., Tirolites cf. cassianus Quenstedt, Tirolites rossicus Kiparisova, Tirolites longilobatus Shevyrev (403-26, -27, $-28,-32,-34$, collected from several beds).

From the lower part:

Pseudosageceras sp., Tirolites cf. cassianus Quenstedt, Tirolites sp. (403-14, -17, -19, -21, -25, collected from several beds)

Pseudosageceras longilobatum Kiparisova, Tirolites cf. cassianus Quenstedt, Tirolites sp. (403-3, -5, collected from different beds)
Brachiopod: Piarorhynchella mangyshlakensis Dagys (404-1); bivalves (404-1); gastropods (404-1)

Bivalves

Small gastropods

From the upper part: bivalves, Neoschizodus cf. laevigata (Zieten), Leda sp. (403-32, -36, collected from different beds); gastropods (403-31) From the lower part: bivalve, Mytilidae gen. et sp. indet. (403-21); gastropods (403-21); amphibian remains (403-21)

Bivalves: Myophoria sp., Ostreidae gen., sp. indet. (403-2, 8 collected from different beds); fish remains (scales) (403-2) Bivalves: Mytilidae, Monotidae (403-1, -4, collected from different beds)

aDorikranites beds, characterized by the conodont Neospathodus cf. brevissimus Orchard (Balini et al. 2000) and rare foraminifera (Vuks 1997), have not been found by us in the block investigated.

bProcarnites sp. (Balini et al. 2000) and "Dinarites" astachovae Gavrilova (Gavrilova 1989) were apparently discovered in the upper and lower parts of Member 17, respectively. ${ }^{c}$ Conodonts Neospathodus sp., Neospathodus sp. C, Neospathodus hommeri (Bender), Neospathodus abruptus Orchard, Neospathodus symmetricus Orchard, Neospathodus dolnapae Nicora (Balini et al. 2000) were apparently found in Member 15.

${ }^{d}$ Conodonts Neospathodus abruptus Orchard, Neospathodus dolnapae Nicora and Neospathodus sp. (Balini et al. 2000) and foraminifera Nodosaria hoae (Trifonova) (Vuks 1997) were apparently discovered in the upper part of Member 9.

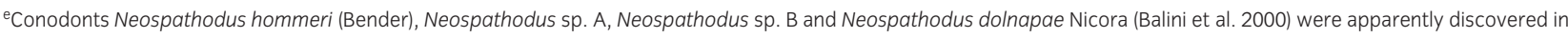
the lower part of Member 7. 


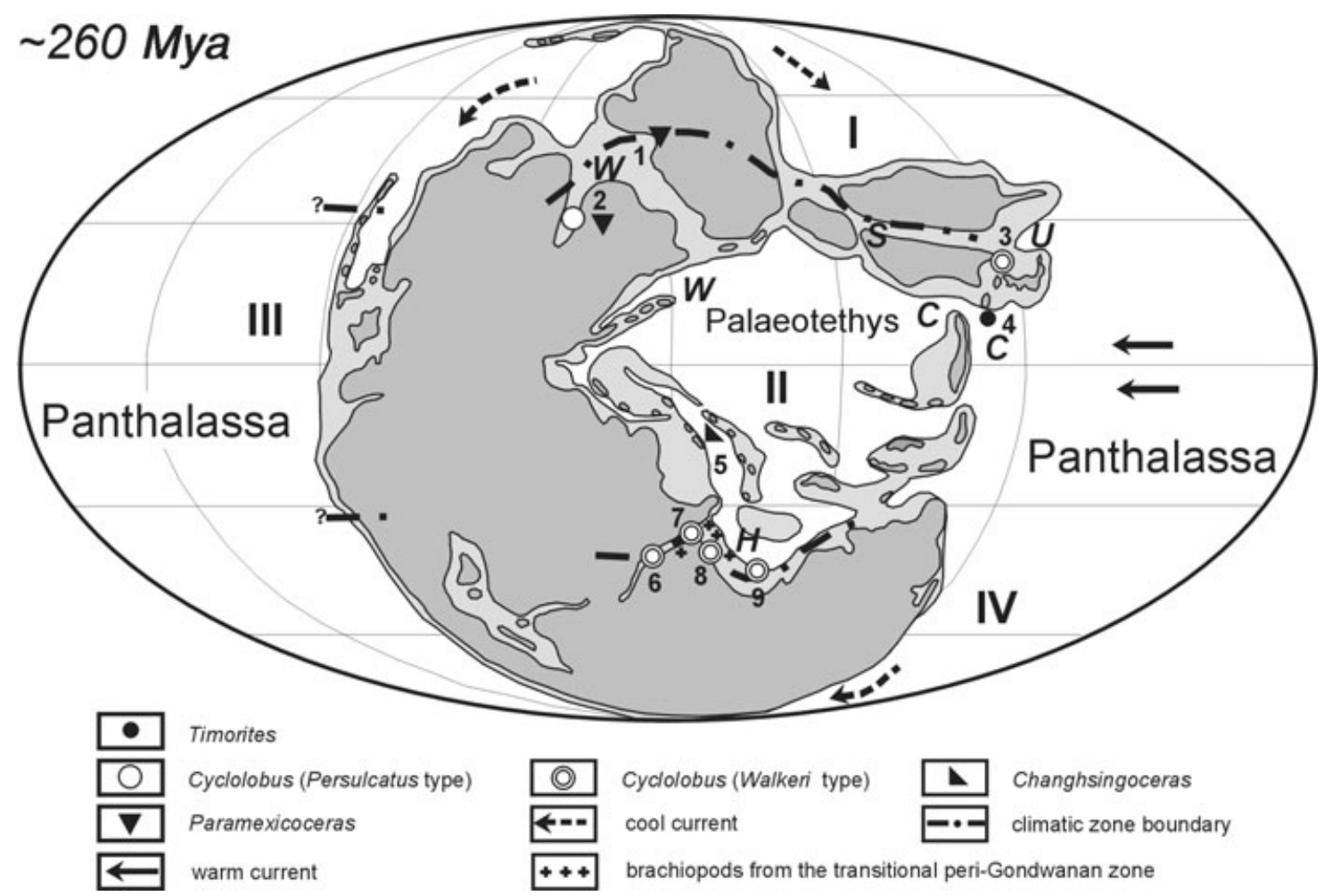

Fig. 5 Expansion of the tropical-subtropical zone during the Early Wuchiapingian (base map after Ziegler et al. 1999): 1, Verkhoyansk area (Popov 1970); 2. East Greenland (Frebold 1932; Nassichuk et al. 1966; Nassichuk 1995); 3, South Primorye (Zaharov 1983); 4, Kitakami (Ehiro \& Bando 1985); 5, Transcaucasia (Zaharov 1985); 6, Madagascar (Furnish \& Glenister 1970); 7, Salt Range (Furnish \& Glenister 1970); 8, central Himalayas (Diener 1903); 9 , Tibet (Leonova 2002). Climatic zones/realms: I, Boreal warm-temperate; II, Tethyan tropical-subtropical; III, American tropical-subtropical; IV, Gondwanan warm-temperate. Provinces: C, Cathasian; H, Himalayan; U, Ussurian; W, western Tethyan.

the upper part of the Paratirolites kittli Zone in Transcaucasia and North Caucasus, and $23.1^{\circ} \mathrm{C}$ for the upper part of the Itomodesma costatum Zone (Zakharov, Biakov et al. 2005), which are confirmed by the data on ammonoid diversity (Fig. 2). Very high palaeotemperatures for the Lopingian age Joulfa section in Iran (23$\left.34^{\circ} \mathrm{C}\right)$ and the Meishan section in South China $\left(26-32^{\circ} \mathrm{C}\right)$ were similarly obtained by Korte, Jasper et al. (2005). Some of these facts seem to be in disagreement with Beauchamp \& Baud's (2002) hypothesis that the northwest margin of Pangea was under the influence of cold to very cold waters for nearly $30 \mathrm{My}$ of the post-Sakmarian Permian, which was the period of chert accumulation in this area.

Late Permian expansions of the tropical-subtropical zone may be linked to both the Early Wuchiapingian and the Late Changhsingian temperature maxima. This is supported by the wide geographical distribution of thermophilous cyclolobid ammonoids (Zakharov \& Ehiro in press) and conodonts (Mei \& Henderson 2001) in the early Wuchiapingian (Fig. 5). Our ammonoid data agree with the data on early Wuchiapingian ammonoids from the Kitakami area, which Ehiro (1997, 1998, 2001b) considered to be similar to ammonoids from the tropical Cathaysian province. Latest Changhsingian ammonoids of South Primorye are especially similar to those from the Cathaysian province (Zakharov \& Oleinikov 1994). The wide distribution of Cathaysian elements may also be explained by latitudinal expansion of the tropical-subtropical zone at that time. The southward invasion of abundant warm-water brachiopods, conodonts, calcareous sponges and gastopods in the Southern Hemisphere during the latest Changhsingian has been confirmed by Shen et al. (2000) and Shen et al. (2006). However, no Changhsingian ammonoids have been discovered at high latitudes (if our assumption concerning the earliest Induan age of all Otoceras and Tompophiceras, and associated Hypophiceras, from the Setorym section, Verkhoyansk area, is correct), which is still unexplainable.

Another possible explanation for the existence of warm conditions in the south Far East during the Late Permian and possibly the Early Triassic is the location of the South Kitakami and South Primorye near the equator. This is 


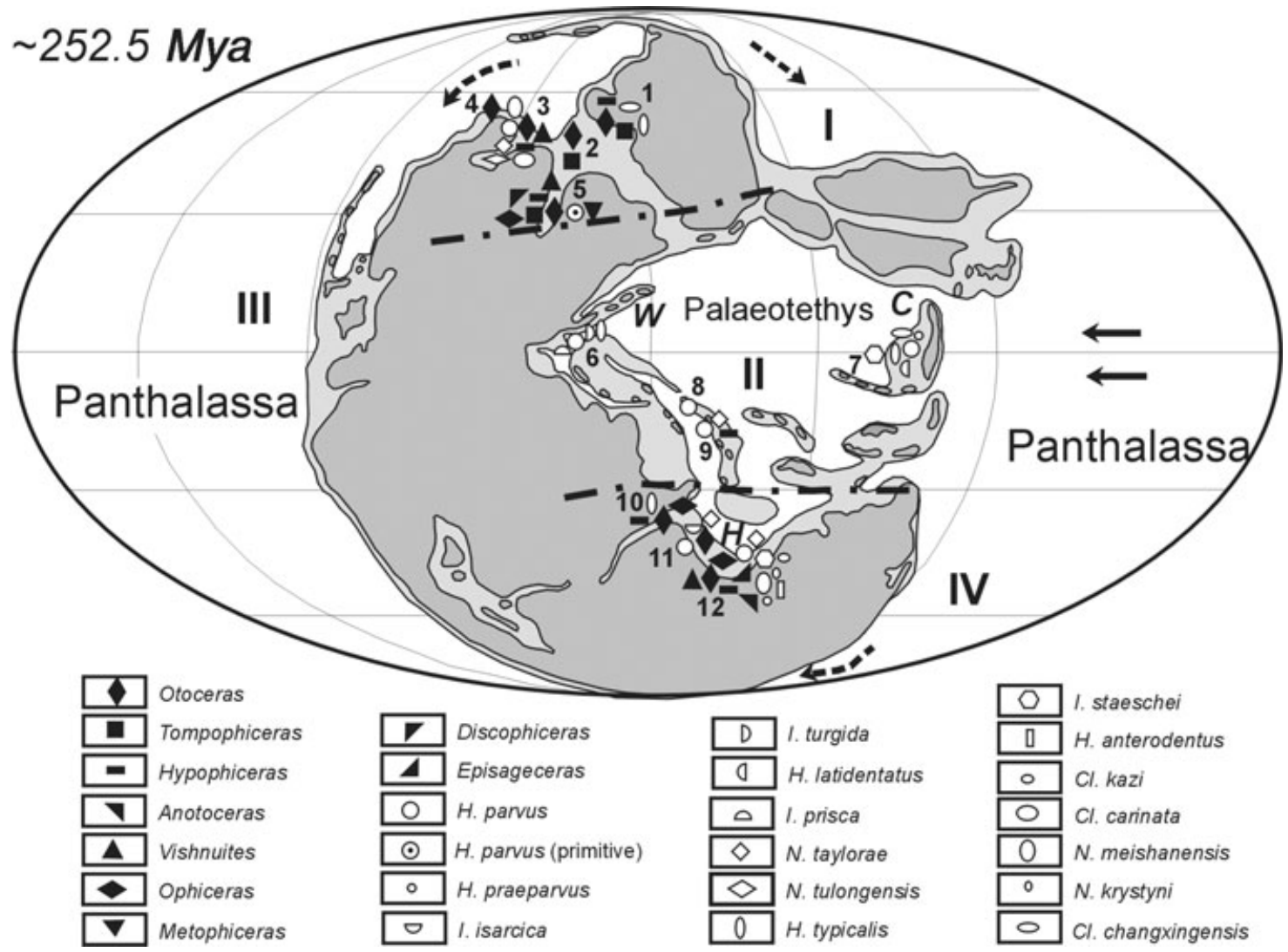

Fig. 6 Reduction of the topical-subtropical zone during the earliest Induan (base map after Ziegler et al. 1999): 1, Verkhoyansk area (Zakharov 2002); 2, Svalbard (Korčinskaja \& Vavilov 1987); 3, Arctic Canada (Orchard 1994; Tozer 1994; Orchard \& Tozer 1997); 4, Alaska (Kummel's data; Zaharov 1968); 5, eastern Greenland (Spath 1930; Ševyrev 2000; Kozur 1998); 6, Alps (Perri \& Farabegoli 2003); 7, South China (Yin et al. 1996; Mei et al. 1998; Mei \& Henderson 2001); 8, Transcaucasia (Zakharov, Biakov et al. 2005); 9, Iran (Bando 1979; Kozur 2004); 10, Kashmir (Nakazawa 1981, 1993; Nakazawa \& Kapoor 1981; Ševyrev 1999); 11, southern Tibet (Jin et al. 1996); 12, Himalayas (Kummel 1972; Orchard \& Krystyn 1998; Ševyrev 1999). Other designations are as listed in Fig. 4. The latest radiometric data (Mundil et al. 2004; Menning et al. 2007) have been used.

based on the palaeomagnetic data from South Primorye (Zaharov \& Sokarev 1991a, b), or on both palaeontological and the aforementioned palaeomagnetic data from the south Far East (Ehiro 1997, 1998, 2001b; Brayard et al. in press). It seems to be debatable because of the strong Early Cretaceous remagnetization of Permian and Triassic rocks in the Russian Far East and Japan, which are used for palaeomagnetic investigation (Kodama, pers. comm.). It is clear that the palaeoposition of South Primorye and Kitakami within a palaeoclimatic zone cannot be determined precisely without having reliable palaeomagnetic data.

The extensive loss of ammonoid species took place during the Permian-Triassic boundary (PTB) ecological crisis. Many hypothetical processes have been proposed to explain PTB events, which have recently been reviewed by Berner (2002), Kidder \& Worsley (2004) and Richoz (2006). A question that remains is the impact of temperature, because there is no information on seawater palaeotemperatures for the PTB beds: no well-preserved fossils suitable for oxygen isotopic investigation have been discovered within this interval. However, data with a bearing on the main trends in temperature changes have been obtained using the $\mathrm{Ca} / \mathrm{Mg}$ ratio method for carbonate sequences. According to Zaharov et al. (2001), the PTB transition in the Karabaglyar-2 and Akhura sections in Transcaucasia (located between low and middle latitudes at the very end of the Changhsingian) shows a $\mathrm{Ca} / \mathrm{Mg}$ ratio in carbonates rising from $170-178$, in the upper part of the Paratirolites kittli Zone, to 185 in the overlying Pleuronodoceras occidentale-Xenodiscus jubilaearis interval, which is interpreted as a gradual temperature drop during the latest Changhsingian. The highest $\mathrm{Ca} / \mathrm{Mg}$ ratio (197-204), reflecting the lowest magnesium content at the PTB in Transcaucasia, falls in interval "a" (Fig. 2), which is a stromatolitic limestone characterized by the first appearance of the conodont $H$. parvus. In overlying limestones of the Isarcicella 


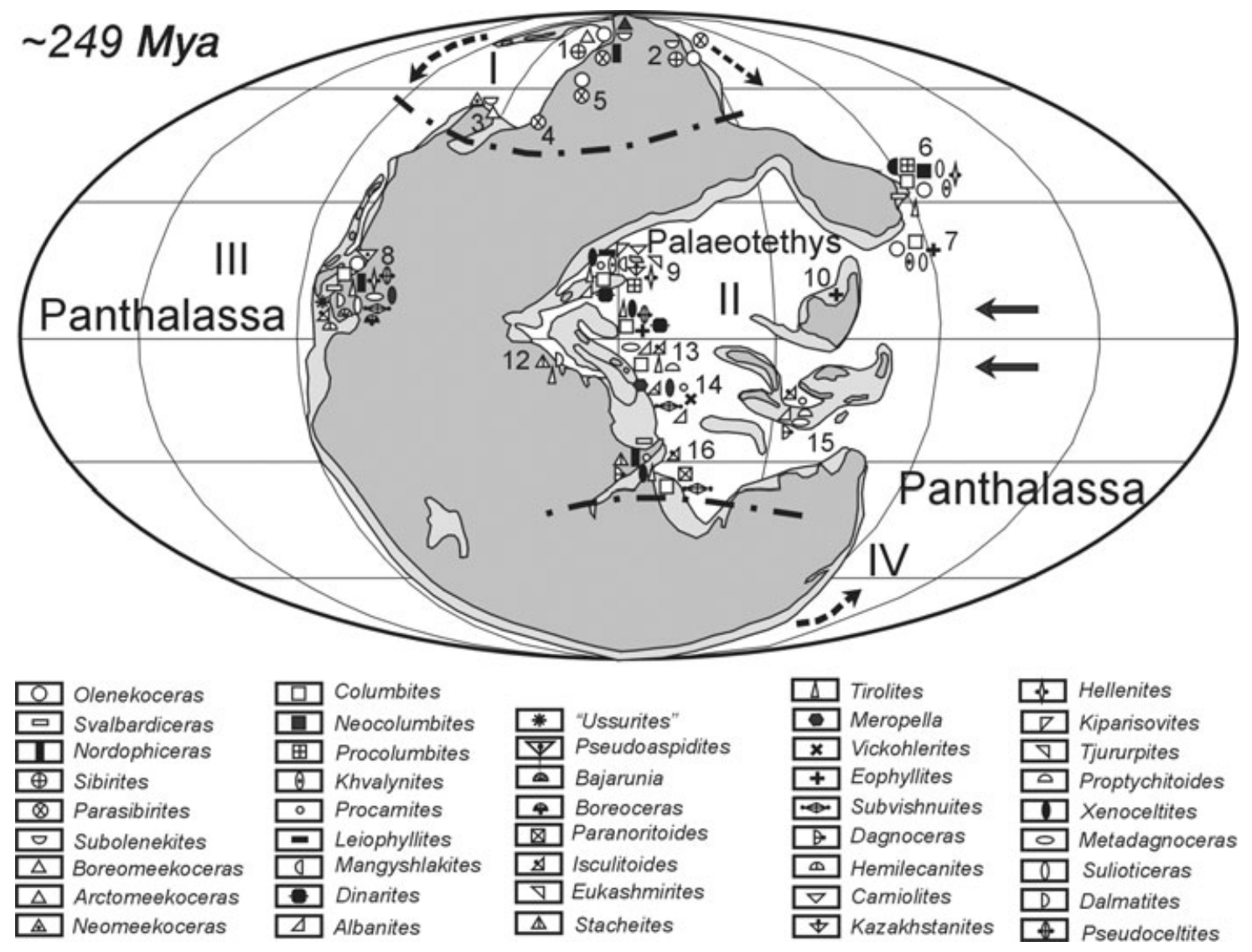

Fig. 7 Expansion of the tropical-subtropical zone during the early part of the late Olenekian Parasibirites grambergi Zone (base map after Ziegler et al. 1999): 1, Arctic Siberia (Dagis \& Ermakova 1988); 2, Kolyma area (Byčkov 1972; Zaharov 1978); 3, Arctic Canada (Tozer 1994); 4, Svalbard (Tozer \& Parker 1968; Korčinskaja 1970); 5, Verkhoyansk area (Zaharov 1978); 6, South Primorye (Zakharov 1997); 7, Kitakami (Bando \& Ehiro 1982); 8, Idaho and Nevada (Kummel 1969); 9, Mangyshlak (Astahova 1960; Ševyrev 1968, 1990; Gavrilova 1980); 10, South China (Tong et al. 2004); 11, North Caucasus (Ševyrev 1990); 12, Alps (Krystyn 1974); 13, Iran (Ševyrev 1990); 14, Afganistan (Kummel 1969); 15, Timor (Kummel 1969); 16, Salt Range (Kummel 1969). Other designations are as listed in Fig. 4. The latest radiometric age by Galfetti, Bucher, Ovtcharova et al. (2007) has been used.

isarcica Zone in the Karabaglyar-2 section (yielding the ammonoid Lythophiceras medium Griesbach), the Ca/Mg ratio is lowered (174-176), reflecting a rise in magnesium content. We interpret the lowest magnesium content as a short-term fall of the palaeo-seawater temperature at the very end of the Changhsingian, and particularly at the beginning of the Induan (FAD $H$. parvus), just after the significant negative carbon isotope excursion (Baud et al. 1989; Zakharov, Biakov et al. 2005). It may also be caused by a fundamental change in PTB sedimentation, as expected by Baud et al. (2007). However, Kozur (2007) discovered a cool-water conodont fauna in the Pleuronodoceras occidentale-Xenodiscus jubilaearis interval of Transcaucasia and Iran (the discovery of volcanic microsphaerulites in this level in Iran, and in the Germanic Basin, documents volcanic activity only occuring during its habitation in cool conditions). These palaeontological and volcanological patterns are consistent with what we propose.
The abrupt decline in heavy carbon isotope concentrations just prior to the PTB (Baud et al. 1989; Magaritz 1989; Yin \& Zhang 1996; Musashi et al. 2001; Korte, Jasper et al. 2005) reflects a global reduction in primary biological productivity (Alcala-Herrera et al. 1992). This might have been induced by increasing oxygen deficiency in response to a dramatic decline in phytoplankton photosynthesis (Hallam 1994). Short-term cooling, following significant warming, and a possible reduction of atmospheric oxygen during the PTB were the likely consequences of the release of $\mathrm{CO}_{2}$ from the Siberian traps (Conaghan et al. 1994; Kozur 2007; Payne \& Kump 2007; Riccardi et al. 2007), and also of methane from seafloor gas hydrates (clathrates) (Martin \& Macdougall 1995; Berner 2002; Kidder \& Worsley 2004; Krull et al. 2004; Payne et al. 2004).

Bando (1973, 1979, 1980) considered that the Dorashamian genus Julfotoceras was most intimately related to Otoceras, the ammonoid index fossil for the 


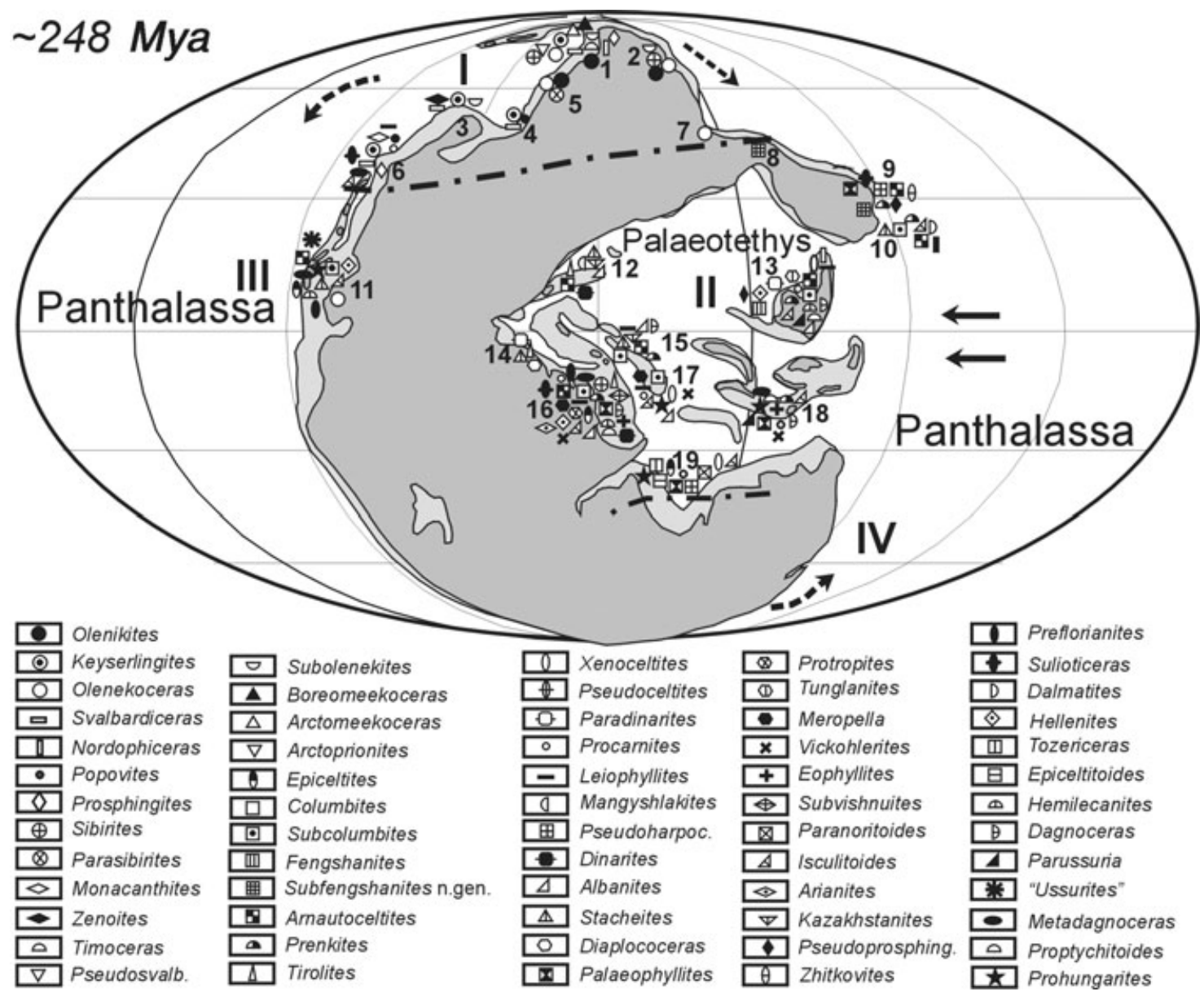

Fig. 8 Reduction of the tropical-subtropical zone during the late part of the late Olenekian Olenikites spiniplicatus Zone (base map after Ziegler et al. 1999): 1, Arctic Siberia (Mojsisovics 1886; Zaharov 1978); 2, Kolyma area (Byčkov 1972; Zaharov 1978); 3, Arctic Canada (Tozer 1994); 4, Svalbard (Korčinskaja 1970; Mørk et al. 1999); 5, Verkhoyansk area (Zaharov 1978); 6, British Columbia (Tozer 1994); 7, Uda River basin, Amur River basin (Ehiro et al. 2006); 8, Bolshiye Churki, Lesser Hingan (Okuneva 1976); 9, South Primorye (Zakharov 1997); 10, Kitakami (Bando \& Shimoyama 1974; Bando \& Ehiro 1982); 11, Nevada (Kummel 1969); 12, Mangyshlak (Astahova 1960; Ševyrev 1968, 2002; Gavrilova 1980, 1989); 13, South China (Chao 1959; Kummel 1969); 14, Alps (Krystyn 1974); 15, Iran (Ševyrev 1990); 16, Chios and Albania (Kummel 1969); 17, Afghanistan (Kummel 1969); 18, Timor (Kummel 1969); 19, Salt Range (Guex 1978). Other designations are as listed in Fig. 4. The latest radiometric age by Lehrmann et al (2006) and Galfetti, Bucher, Ovtcharova et al. (2007) has been used.

PTB beds in both the Himalayas and the Boreal realm. However, our morphological analysis shows that the ancestor of the genus Otoceras apparently originated from the Dzhulfian-Dorashamian Avushoceras, which was an inhabitant of the tropical-subtropical climatic zone (i.e., such as that in Transcaucasia). These two genera (Avushoceras and Otoceras) have very similar suture lines; besides, the juvenile Otoceras shell looks like the adult Avushoceras (Zaharov 1971; Zaharov \& Pavlov 1986a), which reflects a possible anabole in the phylogenetic line AvushocerasOtoceras. We can hypothesize that the ancestral form migrated into higher northern latitudes, following phytoplankton, crustaceans and other organisms, probably to restore its food supply that had been disrupted by short-term cooling, as documented by $\mathrm{Ca} / \mathrm{Mg}$ studies in Permian-Triassic carbonates in Transcaucasia (Zaharov et al. 2001), and possibly the oceanic superanoxia of the Permian-Triassic boundary transition (Hallam 1994; Wignall \& Twitchett 2002), and/or other factors exerting a stronger influence in some low-middle latitude areas.

We suggest that the wide distribution of the cool-water Otoceras faunas at high palaeolatitudes of the Northern Hemisphere (Boreal realm), and at middle palaeolatitudes of the Southern Hemisphere (Himalayan province), reflects the short-lived expansion of the warm-temperate zone, and the favourable reduction of the tropicalsubtropical climatic zone, which would have been a result of the aforementioned short-term cooling that mainly 


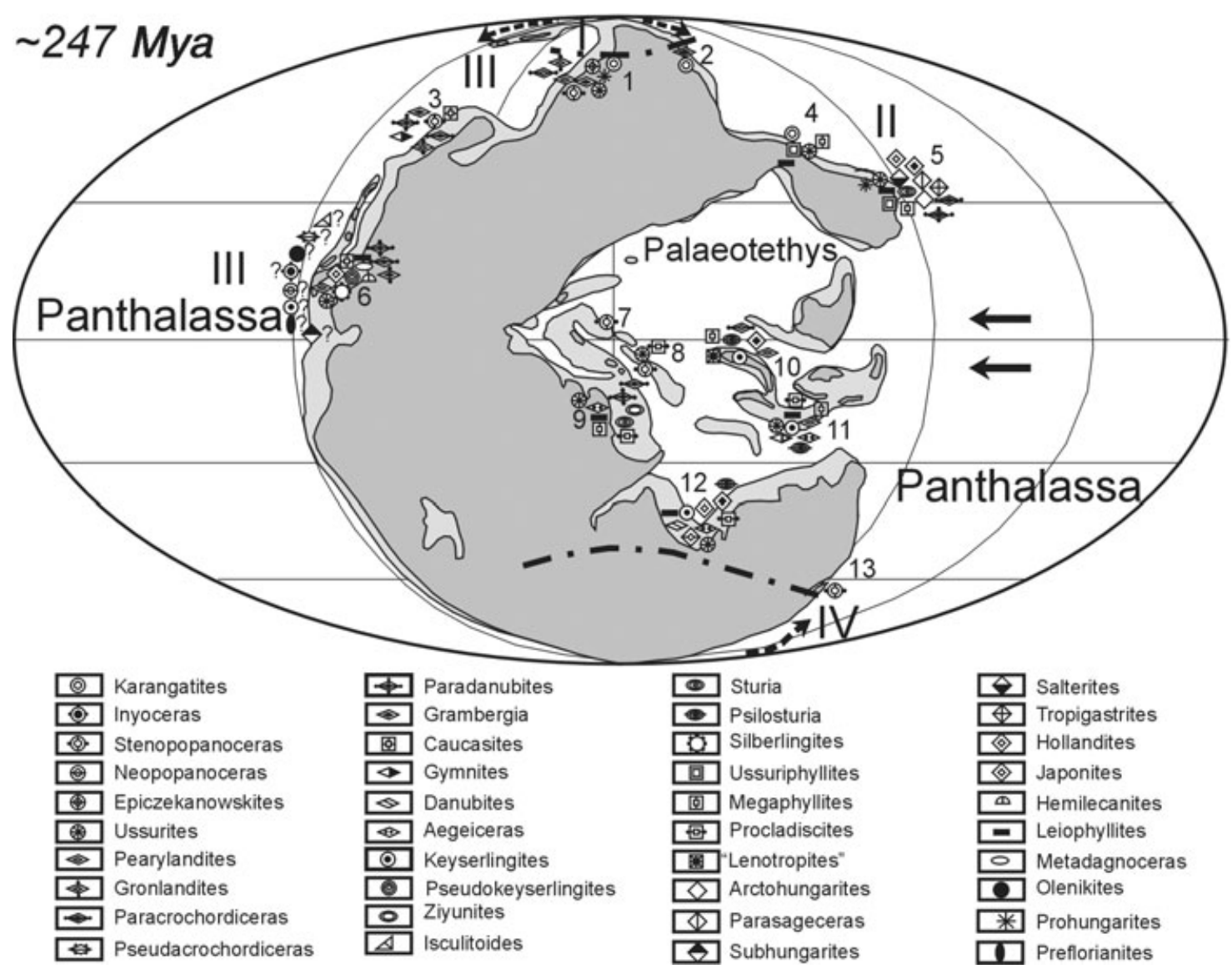

Fig. 9 Expansion of the tropical-subtropical zone during the earliest Anisian Karangatites evolutus beds (base map after Ziegler et al. 1999): 1, Arctic Siberia (Popov 1968; Zaharov 1978); 2, Kolyma area (Byčkov 1972); 3, British Columbia (Tozer 1994); 4, Bolshiye Churki, Lesser Hingan (Okuneva 1976); 5 , South Primorye (Zakharov, Popov et al. 2005); 6, Nevada and Idaho (Bucher 1989; Ševyrev 2002); 7, North Caucasus (Ševyrev 1990); 8, Iran (Ševyrev 1990); 9, Chios (Fantini Sestini 1981; Gaetani et al. 1992); 10, Qinghai (Wang 1985; Bucher 1989); 11, Timor (Welter 1915; Ševyrev 1990); 12, Himalayas (Diener 1895a; Ševyrev 1990); 13, New Zealand (Kummel 1965). Other designations are as listed in Fig. 4. The last radiometric age by Lehrmann et al. (2006) has been used for the base of the Anisian.

occurred at the very beginning of the Induan (FAD Hindeodus parvus) (Fig. 6).

According to Brayard et al.'s (2006) calculation, no latitudinal or longitudinal ammonoid generic richness gradient can be recognized during early Induan (Griesbachian) time, but a weak latitudinal diversity gradient emerges during late Induan (Dienerian). In contrast, the Olenekian is characterized by a stronger latitudinal diversity gradient, showing a less uniform climate tendency during the Early Triassic.

According to our oxygen isotope temperature determinations (Zakharov et al. 1999), late Olenekian and late Anisian climates in Arctic Siberia seem to be about 7.4 and $6.6^{\circ} \mathrm{C}$ warmer, respectively, than early Olenekian temperatures. The calculated middle-late Anisian isotopic palaeo-seawater temperatures of about $15^{\circ} \mathrm{C}$ for Arctic Siberia (Kurušin \& Zaharov 1995; Zakharov et al. 1999) approach those of the late Olenekian at about $16.2^{\circ} \mathrm{C}$ (Zakharov et al. 1999). No oxygen isotopic palaeotemperature data have been obtained for other levels of the Lower-Middle Triassic.

Additional information on Early-Middle Triassic marine environments may be obtained from data on carbon isotopic anomalies. As suggested by AlcalaHerrera et al. (1992), some variations in the ${ }^{13} \mathrm{C} /{ }^{12} \mathrm{C}$ ratios recorded in deep-water marine carbonates might be controlled by the carbon budget, upwelling and primary productivity. It is difficult to separate the effect of each of these factors in deep-water conditions, but when worldwide carbon isotope shifts are observed in shallow-water carbonates they can generally be attributed to changes in primary biological productivity, most likely in the phytoplankton. The peculiarities in the distribution of phytoplankton, one of the main organisms that utilize solar energy in today's oceans, have been thoroughly investigated (Bogorov 1974). As photosynthesizers, 
phytoplankton are concentrated in the upper $100 \mathrm{~m}$ of the water column, but their geographical distribution depend on the degree of hydrological intermixing of water under the influence of thermal gradients and winds. Phytoplankton productivity is high in areas characterized by an intensive vertical circulation, such as in upwelling areas. The small numbers of plankton in recent Arctic and Antarctic seas is probably connected with the short summer growth interval of phytoplankton at high latitudes. However, during times when polar ice was absent, the hydrological conditions were probably considerably different from those of the present day, in that poleward transport of large equatorial warm-water masses and weaker vertical circulation of waters probably occurred in some climatic zones. Therefore, the actual causes of Phanerozoic carbon isotopic anomalies can only be evaluated under conditions of poor constraints. Our data show that the positive $\delta^{13} \mathrm{C}$ anomalies at different levels of the Permo-Triassic younger than the Sakmarian are usually characterized by a high Mg content (Zaharov et al. 2001). These seem to be mainly related to times of high biological productivity in the ocean basins, caused by conditions stimulated by transgressions and warm climate.

The carbon isotope and $\mathrm{Ca} / \mathrm{Mg}$ data from Transcaucasia, North Caucasus, South Primorye, South China, Iran, Pakistan, Oman and the Alps suggest a recurrent warming trend, beginning in the latest parts of the early Induan (Lythophiceras medium Zone) (Zakharov et al. 2000), and continuing into Anisian times, reaching temperature maxima in the Middle-Late Induan (Zakharov, Biakov et al. 2005), early Olenekian (Richoz 2006; Horacek \& Abart 2007), Middle Olenekian (TirolitesAmphistephanites Zone) (Zakharov et al. 2000; Korte, Kozur et al. 2005; Richoz 2006; Lehrmann et al. 2006; Galfetti, Bucher, Brayard et al. 2007; Galfetti, Bucher, Ovtcharova et al. 2007; Horacek \& Abart 2007) and in the beginning of the Anisian (Zakharov et al. 2000; Korte, Kozur et al. 2005; Richoz 2006). Hence, it is anticipated that there was a general reduction (in latitudinal extent) of the warm-temperate climatic zone throughout the late Induan to earliest Anisian (Figs. 7, 9), which more or less agrees with data on Early Triassic-earliest Anisian ammonoid and brachiopod diversity (Figs. 3, 4) (Xu \& Lui 1983; Brayard et al. 2006; Brayard et al. 2007; Zaharov et al. 2008).

In the middle and earliest part of the late Olenekian, as in the early Wuchiapingian and late Changhsingian, the tropical-subtropical climatic zone was very extensive. Typical Tethyan Tirolites faunas appeared in the middle Olenekian, approximately 2 Mya, after the PTB mass extinction (Ovtcharova et al. 2006), and continued to play a significant role in the earliest part of the late Olenekian ammonoid assemblages in the western Tethys.
Ammonoids of Tethyan type inhabited a tropicalsubtropical climatic zone in the earliest part of the late Olenekian, and were very abundant and diversified: among them, columbitid ammonoids seem to be one of main indicators of the tropical-subtropical climatic zone for that time (Fig. 4). Subolenekites, Olenekoceras, Svalbardiceras, Nordophiceras, Parasibirites, Sibirites, Boreomeekoceras, Arctomeekoceras and Neomeekoceras are usually characteristic of the northern warm-temperate climatic zone (Boreal realm) in the earliest part of the late Olenekian, but some of them (Olenekoceras, Nordophiceras and Svalbardiceras) may possibly have migrated southwards along the eastern and western areas of Pangea, to middle-latitude (South Primorye and Kitakami) and low-latitude (Idaho and Nevada) areas of the Northern Hemisphere. Three ammonoid genera common in Boreal assemblages (Nordophiceras, Svalbardiceras and Arctomeekoceras) have been discovered in the middle latitudes of the Southern Hemisphere (i.e., the Salt Range) (Kummel 1969), in association with typical Tethyan ammonoids Tirolites, Columbites and Procolumbites.

The data on the geographical distribution of ammonoids of the latest part of the late Olenekian show that cooling apparently took place (in the Subfengshanites multiformis Zone) just before the earliest Anisian climatic optimum, initiating expansion of the warmtemperate climatic zone southwards in the Northern Hemisphere. The southern boundary of the Boreal realm reached the northern part of the Khabarovsk area in the east, and the southern part of British Columbia in the west (Fig. 8). Abundant endemic ammonoid elements, such as Olenikites, Olenekoceras, Keyserlingites, Nordophiceras, Svalbardiceras, Prosphingites, Popovites, Sibirites, Parasibirites and Monocanthites were present in the Boreal realm in the latest part of the late Olenekian, showing the lack of any remarkable faunistic relations between the Tethys and the Boreal realm at that time.

Fossiliferous sedimentary rocks of earliest Anisian are comparatively restricted in distribution in Eurasia, and their global correlation is still uncertain. (Following Hyatt \& Smith's [1905] original proposal, we continue to consider that the Neopopanoceras haugi Zone in North America is earliest Anisian, in spite of the recent tendency to expect it to be latest Olenekian [Bucher 1989; Tozer 1994; Ševyrev 2000].) However, faunal connections between the Boreal realm and Tethys (via the distribution of juvenile invertebrate individuals, possibly by oceanic currents) were undoubtedly more active in the early Anisian compared with the late Olenekian, possibly because of the extension of a more homogeneous climate at the beginning of the Middle Triassic. The existence of the earliest Anisian (Ussuriphyllites amurensis Zone) temperature maximum is confirmed by the wide latitu- 

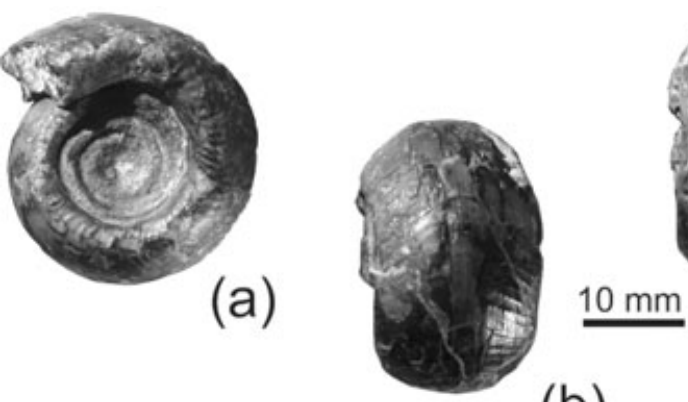

(b)

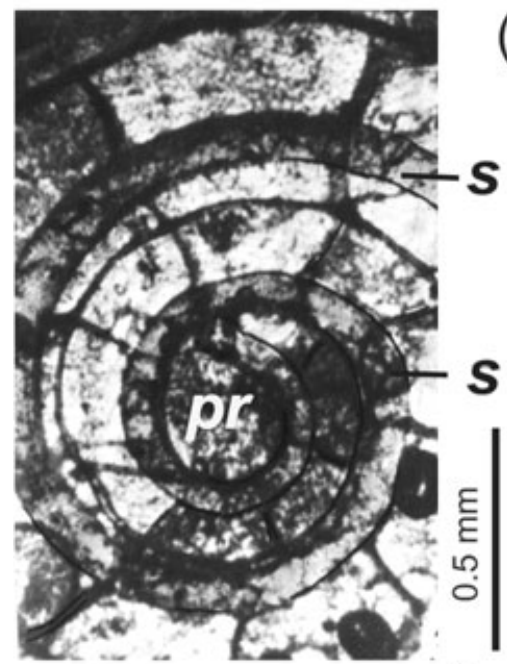

(d)
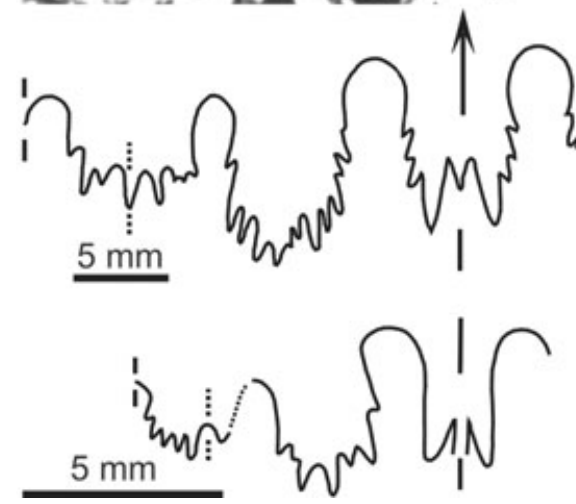

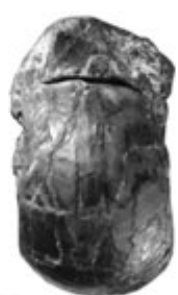

(c)

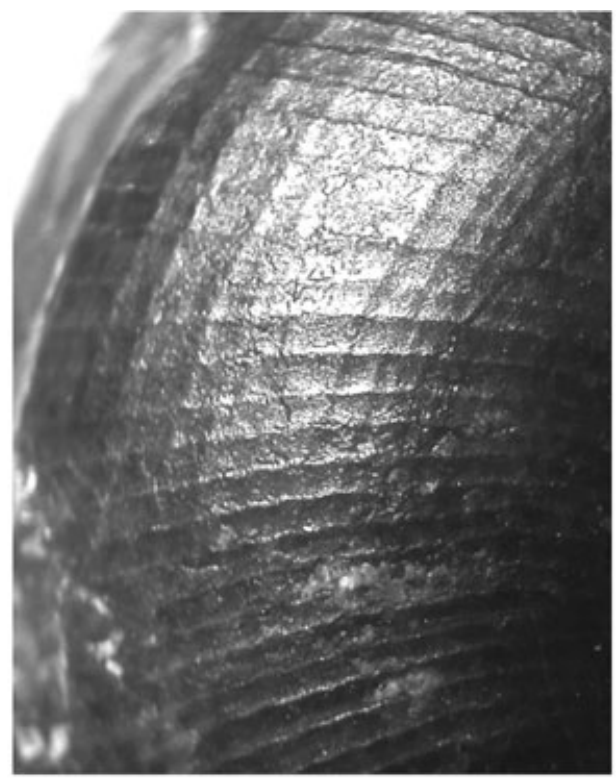

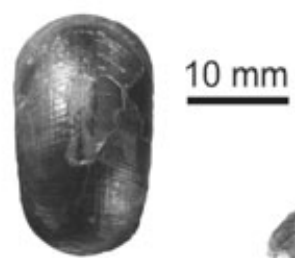

(f)

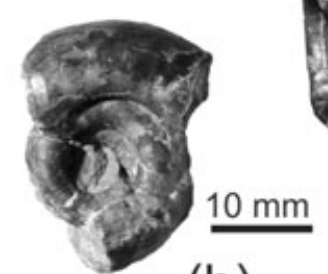

(h)

(k)

(I)

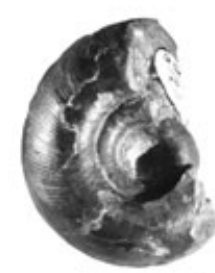

(e)

$1 \mathrm{~mm}$

(g)

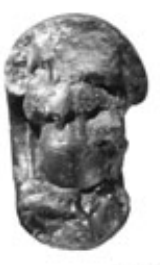

(i)

(j)
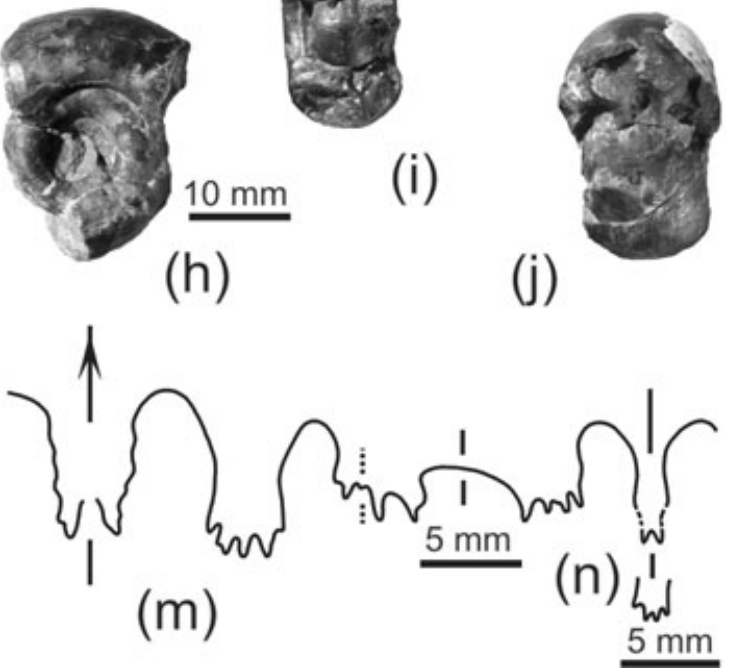

Fig. 10 Subfengshanites multiformis (Kiparisova). (a-c) Dal'nevostočnyj Geologičeskij Institut (Far Eastern Geological Institute) (DVGI) 449/801: lateral, ventral and apertural views. (d) DVGI 71/801: inner shell structure, showing dorsal position of siphuncle at the first whorl, and its subdorasal position at the second whorl (pr, protoconch; s, siphuncle). (e and f) DVGI 452/801: lateral and ventral views. (g) DVGI 452/801: reticulate ornamentation. (h-j) DVGI 451/801: lateral, ventral and apertural views. (k-n) Suture lines. (k and I) VSEGEI 126/5504 (holotype). (k) Height 10 mm; width 18.9 mm. (l) Height 4.8 mm; width 13.6 mm. (n) DVGI 456/801, dorsal lobe: height 6.8 mm; width $16 \mathrm{~mm}$. Upper Olenekian, the uppermost part of the Zhitkov Cape Formation. Samples 126/5504 (holotype), 71/801, 449/801, 451/801, 452/801 and 456/801 were collected from the lens of calcareous marl, $0.3 \mathrm{~m}$ thick, located at the base of the Subfengshanites multiformisone Zone, Russian Island, Zhitkov Cape.

dinal extent of the tropical-subtropical climatic zone (Fig. 9). Many earliest Anisian ammonoids, such as Stenopopanoceras, Karangatites, Prohungarites, Pearylandites, Paracrochordiceras, Paradanubites and Grambergia are cosmopolitan, being common in the tropical-subtropical climatic zone in low, middle and high latitudes. However, earliest Anisian ammonoids as a whole were less diverse at high latitudes: among the earliest Anisian endemics of high latitudes, only forms of Gronlandites are known. Typical late Olenekian ammonoid elements of the Boreal realm, such as Keyserlingites, disappeared before the earliest Anisian assemblages at high latitudes because they 
apparently migrated to the middle and low latitudes in both hemispheres (Nevada, Timor, Tibet, Himalayas and possibly Primorye). Their unchecked advance, similar to the global migration of Stenopopanoceras and some 18 other ammonoid genera, may be explained by a warm and uniform climate during the earliest Anisian.

\section{Conclusions}

Correlation of palaeontological and geochemical data suggests that most evident thermal maxima existed during the late Kungurian, early Wuchiapingian, latest Changhsingian, middle Olenekian and earliest Anisian. Successive latitudinal expansions and reductions of the tropical-subtropical zone during the Late Permian, and the Early and Middle Triassic, are a result of significant climatic fluctuations. The wide distribution of the coolwater Otoceras faunas at high palaeolatitudes of the Northern Hemisphere (Boreal realm), and at middle palaeolatitudes of the Southern Hemisphere (Himalayan province), reflecting the short-lived expansion of the warm-temperate and the favourable reduction of the tropical-subtropical climatic zones, seems to be a result of short-term cooling at the very beginning of the Induan. In contrast, the unprecedented expansion of the tropicalsubtropical climatic zone to higher latitudes at the beginning of the Anisian suggests that the earliest Anisian was a time of stable and warm climatic conditions.

\section{Description of a new genus}

Superfamily Columbitoidea Spath, 1930

[nom. transl. Zaharov, 1978 (ex Columbitidae Spath, 1930)]

Family Columbitidae Spath, 1930

Genus Subfengshanites gen. nov.

Type species. Subcolumbites multiformis Kiparisova (Voinova et al. 1947: 144, pl. 32, figs. 8-11; Kiparisova 1961: 121, pl. 27, figs. 1-7; Zaharov 1968: 108, pl. 21, figs. 1-3); Upper Olenekian, Subfengshanites multiformis Zone, South Primorye.

Diagnosis. Semi-evolute to evolute cadicone, with broadly arched veter without any tendency of carination, but characterized by marked development of reticulate ornamentation; position of the sifuncle in the first two whorls is dorsal to subdorsal (Fig. 10a-j). Suture line consists of five lobes: $\left(\mathrm{V}_{1} \mathrm{~V}_{1}\right) \mathrm{UU}^{1}: \mathrm{I}\left(\mathrm{D}_{1} \mathrm{D}_{1}\right)$ (Fig. 10k-m). Lobes $U, \mathrm{U}^{1}$ and $\mathrm{I}$ are broad and significantly denticulated at the base. Dorsal lobe has tendency to become a fourindented lobe (Fig. 10n).
Occurrence. Uppermost Olenekian, South Primorye.

Species composition. Single species (Subfengshanites multiformis [Kiparisova] = "Subcolumbites" solites Kiparisova and "Subcolumbites" anomalus Kiparisova) from the upper Olenekian of South Pimorye.

Comparison. The new genus is identified by the most complicated suture among known columbitid ammonoids. Denticulated head-like lateral saddles differentiate it from Fengshanites Chao. A broadly arched venter without any tendency of carination, significantly stronger spiral striae and denticulated lateral lobes differentiate it from Subcolumbites Spath. A broadly arched venter, significantly stronger spiral striae, denticulated head-like lateral saddles and dorsal to subdorsal position of the siphuncle in the second whorl differentiate it from Columbites Hyatt and Smith (Columbites is characterized by its subdorsal to central position in the same stage; Zaharov 1978).

\section{Acknowledgements}

We extend our gratitude to Dr M. Hounslow (UK), Prof. M. Ehiro (Sendai) and anonymous reviewers for providing valuable editorial comments that substantially improved this paper. For help in finding references, we are indebted to Drs M. Balini (Milan), Y. Shigeta (Tokyo) and Ja. Vuks (St. Petersburg). This research was carried out with the financial support of RFBR grant 08-05-00100-a and FEB RAS grant 07-III-B-08-075, Russia. This is a contribution to the IGCP572 project Permian-Triassic Ecosystems.

\section{References}

Abich H.W. 1878. Geologische Forschungen in den Kaukasischen Ländern. Th. 1. Eine Bergkalk-fauna aus der Araxes-Enge bei Djoulfa in Armenien. (Geological investigation in the Caucasus area. Pt. 1 Limestone Mount fauna from the Djulfa canyon in Armenia.) Vienna: Alfred Hölder.

Alcala-Herrera J.A., Grossman E.L. \& Gartner S. 1992. Nannofossils diversity and equitability and fine-fraction $\mathrm{d}^{13} \mathrm{C}$ across the Cretaceous/Tertiary boundary at Walvis Ridge Leg 74, South Atlantic. Marine Micropaleontology 20, 77-88.

Angiolini L., Darbyshire D.P.F., Leng M.J., Stephenson M.H., Brewer T.S., Berra F. \& Jadoul F. 2006. Evidence of climate change from brachiopods within the Lower Permian of Oman. Paper presented at the Second International Palaeontological Congress, 17-21 June, Beijing, China. Astahova [Astachova] T.V. 1960. Novye rannetriasovye ceratity Mangyšlaka. (New Early Triassic ceratites of Mangyshlak.) In B.P. Markovskij (ed.): Novye vidy drevnih rastenij i bespozvonočnyh SSSR 2. (New species of fossil plants and invertebrates of the USSR 2.) Pp. 139-159. Moscow: Gosgeoltekhizdat. 
Bajarunas M.V. 1936. Vozrast cloev c Doricranites (Age of the Doricranites beds.) Izvestija Akademii Nauk SSSR, Serija Geologičeskaja 4, 539-546.

Balini M., Gavrilova V.A. \& Nicora A. 2000. Biostratigraphical revision of the classic Lower Triassic Dolnapa section (Mangyshlak, west Kazakhstan). Zentralblat für Geologie und Paläontologie, Teil I (1998), 1441-1462.

Bando Y. 1973. On the Otoceratidae and Ophiceratidae. Science Report of the Tohoku University, Series 2 (Geology), Special Vol. 6, 337-351.

Bando Y. 1979. Upper Permian and Lower Triassic ammonoids from Abadeh, central Iran. Memoirs of the Faculty of Education, Kagawa University, Part 2, 29, 103-136.

Bando Y. 1980. On the Otoceratacean ammonoids in the central Tethys with a note on their evolution and migration. Memoirs of the Faculty of Education, Kagawa University, Part 2, 30, 23-149.

Bando Y. \& Ehiro M. 1982. On some Lower Triassic ammonites from the Osawa formation at Asadanuki, Towa-cho, Tome-gun, Miyagi Prefecture, Northeast Japan. Transactions and Proceedings of the Palaeontological Society of Japan, New Series 127, 375-385.

Bando Y. \& Shimoyama S. 1974. Late Scythian ammonoids from the Kitakami Massif. Transactions and Proceedings of the Palaeontological Society of Japan, New Series 94, 293-312.

Baud A.M., Magaritz M. \& Holser W.T. 1989. Permian-Triassic of the Tethys: carbon isotope stratigraphy. Geologische Rundschau 78, 649-677.

Baud A., Richoz S. \& Pruss S. 2007. The Lower Triassic anachronistic carbonate facies in space and time. Global and Planetary Change 55, 81-89.

Beachamp B. \& Baud A. 2002. Growth and demise of Permian biogenic chert along northwest Pangea: evidence for end-Permian collapse of thermohaline circulation. Palaeogeography, Palaeoclimatology, Palaeoeology 184, 37-63.

Beatty T.W., Henderson C.M., Orchard M.J. \& Beauchamp B. 2006. Early Triassic (Induan) conodont biostratigraphy of the Sverdrup Basin, Canadian Arctic. Boreal Triassic 2006. Longyearbyen, Svalbard, 16-19 August 2006. NGF Abstracts and Proceedings of the Geological Society of Norway 3, 41.

Berner R.A. 2002. Examination of hypotheses for the Permo-Triassic boundary extinction by carbon cycle modeling. Proceedings of the National Academy of Sciences of the United States of America 99, 4172-4177.

Bogorov V.G. 1974. Plankton Mirovogo okeana. (Plankton of the World Ocean.) Moscow: Nauka.

Brayard A. \& Bucher H. 2008. Smithian (Early Triassic) ammonoid faunas from northwestern Guangxi South China): taxonomy and biochronology. Fossils and Strata 55. Copenhagen: Wiley-Blackwell.

Brayard A., Bucher H., Escarguel G., Fluteau F., Bourquin S. \& Galfetti T. 2006. The Early Triassic ammonoid recovery: palaeoclimatic significance of diversity gradients. Palaeogeography, Palaeoclimatology, Palaeoecology 239, 374-395.
Brayard A., Escarguel G. \& Bucher H. 2007. The biogeography of Early Triassic ammonoid faunas: clusters, gradients, and networks. Geobios 40, 749-765.

Brayard A., Escarguel G., Bucher H. \& Bruhwiller T. in press. Smithian and Spathian (Early Triassic) ammonoid assemblages from terranes: paleoceanographic and paleogeographic implications. Journal of Asian Earth Sciences.

Brühwiler T., Bucher H., Goudemand N. \& Brayard A. 2007. Smithian (Early Triassic) ammonoids successions of the Tethys: new preliminary results from Tibet, India, Pakistan and Oman. In S.G. Lucas \& J.A. Spielmann (eds.): The global Triassic. New Mexico Museum of Natural History and Science Bulletin 41, 25-26.

Buch L. 1831. Explication de trois planches d'ammonites. (Explanation of the three ammonite plates.) Paris. Reproduced in Leopold von Buch's Gessamelte Schriften. Pp. 94-99. Berlin: Georg Reimer.

Bucher H. 1989. Lower Anisian ammonoids from the northern Humboldt Range (northwestern Nevada, USA) and their bearing upon the Lower-Middle Triassic Boundary. Eclogae Geologicae Helvetiae 82, 945-1002.

Burij I.V. 1959. Stratigrafija triasovyh otloženij Južnogo Primor'ja. (Stratigraphy of Triassic sediments of South Primorye.) Trudy Dal'nevostočnogo Politehničeskogo Instituta 54, 3-34.

Byčkov Ju.M. [Bytchkov Y.M.] 1972. Nižnij trias verhov'ev r. Kulu. (Lower Triassic of the upper Kulu River.) In I.E. Drapkin (ed.): Materialy po geologii i poleznym iskopaemym Severo-Vostoka SSSR 20. (Data on geology and minerals of the north-east USSR 20.) Pp. 78-82. Magadan: Magadanskoe Knižnoe Izdatel'stvo.

Chao K. 1959. Lower Triassic ammonoids from western Kwangsi, China. Palaeontologia Sinica 9. Peking: Science Press.

Clements F.E. 1916. Plant succession: an analysis of the development of vegetation. Washington D.C.: Carnegie Institute of Washington.

Clements F.E. 1936. Nature and structure of the climax. Journal of Ecology 24, 252-284.

Conaghan P.J., Shaw S.E. \& Veevers J.J. 1994. Sedimentary evidence of the Permian/Triassic global crisis induced by the Siberian hotspot. In A.F. Embry et al. (eds.): Pangea: global environments and resources. Pp. 785-795. Calgary: Canadian Society of Petroleum Geologists.

Čumakov (Chumakov) N.M. 2004. Klimat iklimatičeskie zony permi i rannego triasa. (Climate and climatic zones of the Permian and Early Triassic.) In M.A. Semihatov $\delta$ N.M. Cumakov (eds.): Klimat v epohi krupnyh biosfernyh perestroek. (Climate in the epochs of major biospheric transformations.) Trudy Geologičeskogo Instituta Rossijskoj Akademii Nauk 550, 230-258.

Dagis (Dagys) A.S. \& Ermakova S.P. 1988. Boreal'nye pozdneolenekskie ammonoidei. (Boreal Late Olenekian ammonoids.) Trudy Instituta Geologii i Geofiziki Sibirskogo Otdelenija Akademii Nauk SSSR 714. Moscow: Nauka.

Dagys A.S. 1988. Major features of the geographic differentiation of Triassic ammonoids. In J. Wiedmann \& J. 
Kullmann (eds.): Cephalopods_present and past. Pp. 341-349. Stuttgart: Schweizerbart'sche Verlagsbuchhandlung.

Dagys A. \& Ermakova S. 1996. Induan (Triassic) ammonoids from north-eastern Asia. Revista di Palaeobiologia 15, 401-447.

Diener C. 1895a. The cephalopoda of the Muschelkalk. Memoirs of the Geological Survey of India. Paleontologia Indica, Series 15,

5(2). Calcutta: Geological Survey Office.

Diener C. 1895b. Triadische Cephalopodenfaunen der ostsibirischen. (Triassic cephalopod fauna of the East Siberian marine province.) Mémoires du Comité Geologique 14(3), 1-59.

Diener C. 1897. Himalayan fossils. The Cephalopoda of the Lower Trias. Memoirs of the Geological Survey of India. Palaeontologia Indica, Series 15, 2(1). Calcutta: Geological Survey Office.

Diener C. 1903. Permian fossils of the central Himalayas. Memoirs of the Geological Survey of India, Palaeontologia Indica, Series 15, 1(5). Calcutta: Geological Survey Office.

Diener C. 1916. Die marinen Reiche der Triasperiod. (Triassic marine realm.) Denkschriften kaiserlichen Akademie der Wissenschaften in Wien. Mathematische-naturwissenschaftliche Klasse 93. Vienna: Academy of Sciences.

Ehiro M. 1995. Cephalopod fauna of the Nakadaira Formation (Lower Permian) in the Southern Kitakami Massif, northeast Japan. Transactions and Proceedings of the Palaeontological Society of Japan, New Series 179, 184-192.

Ehiro M. 1997. Ammonoid paleobiogeography of the South Kitakami Palaeoland and palaeogeography of Eastern Asia during Permian to Triassic time. In Y. Jin and D. Dineley (eds.): Proceedings of the 30th International Geological Congress. Vol. 12. Palaeontology and historical geology. Pp. 18-28. Utrecht: VSP.

Ehiro M. 1998. Permian ammonoid fauna of the Kitakami Massif, northeast Japan-biostratigraphy and paleobiogeography. In Y. Jin et al. (eds.): Permian stratigraphy, environments and resources. Vol. 2. Palaeoworld 9, 113-122.

Ehiro M. 2001a. Some additional Wuchiapingian (Late Permian) ammonoids from the Southern Kitakami Massif, northeast Japan. Paleontological Research 5, 111-114.

Ehiro M. 2001b. Origins and drift histories of some microcontinents distributed in the eastern margin of Asian continent. Earth Science 55, 71-81.

Ehiro M. \& Araki H. 1997. Permian cephalopods of Kurosawa, Kesennuma City in the southern Kitakami Massif, north Japan. Paleontological Research 1, 55-66.

Ehiro M. \& Bando Y. 1985. Late Permian ammonoids from the southern Kitakami Massif, northeast Japan. Transactions and Proceedings of the Palaeontological Society of Japan, New Series 137, 25-49.

Ehiro M. \& Misaki A. 2005. Middle Permian ammonoids from the Kamiyasse-Imo district in the southern Kitakami Massif, northeast Japan. Paleontological Research 9, 1-14.

Ehiro M., Shimoyama S. \& Murata M. 1986. Some Permian Cyclolobaceae from the Southern Kitakami Massif, northeast Japan. Transactions and Proceedings of the Palaeontological Society of Japan, New Series 142, 400-408.
Ehiro M., Zakharov Y.D. \& Minjin C. 2006. Early Triassic (Olenekian) ammonoids from Khentey Province, Mongolia, and their paleobiogeographic significance. Bulletin of the Tohoku University Museum 5, 83-97.

Fantini Sestini N. 1981. Lower Anisian (Aegean) ammonites from Chios island (Greece). Rivista Italiana di Paleonologia e Stratigrafia 87, 41-66.

Frebold H. 1932. Marines Unterperm in Ostgroendland und die Frage der Grenzziehung zwischen dem pelagischen Oberkarbon und Unterperm. (Marine Lower Permian in eastern Greenland and problem of the boundary between the pelagian Caroniferous and Permian.) Meddelelser om Grønland 84(4). Pp. 1-35. Copenhagen: Commission for Scientific Research in Greenland.

Furnish W.M. \& Glenister B.F. 1970. Permian ammonoid Cyclolobus from the Salt Range, west Pakistan. In B. Kummel \& C. Teichert (eds.): Stratigraphic boundary problems: Permian and Triassic of west Pakistan. Special Publication 4. Pp. 153-175. Lawrence, KS: Paleontological Institute, University of Kansas.

Gaetani M., Jacobshagen V., Nicora A., Kauffmann G., Tselepidis V., Fantini Sestini N., Mortmann D. \& Skourtsis-Coroneou V. 1992. The Early-Middle Triassic boundary at Chios (Greece). Rivista Italiana di Paleonologia e Stratigrafia 98, 181-204.

Galfetti T., Bucher H., Brayard A., Hochuli P.A., Weissert H., Guodun K., Altudorei V. \& Guex J. 2007. Late Early Triassic climate change: insights from carbonate carbon isotopes, sedimentary evolution and ammonoid paleobiogeography. Palaeogeography, Palaeoclimatology, Palaeoecology 243, 394-411.

Galfetti T., Bucher H., Ovtcharova M., Schaltegger U., Brayard A., Brühwiller T., Goudemand N., Weissert H., Hochuli P.A. \& Guodun F.C.K. 2007. Timing of the Early Triassic carbon cycle perturbations inferred from new $\mathrm{U}-\mathrm{Pb}$ ages and ammonoid biochronozones. Earth and Planetary Science Letters 258, 593-604.

Galfetti T., Hochuli P.A., Brayard A., Bucher H., Weissert H. \& Vigran J.O. 2007. Smithian-Spathian boundary event: evidence for global climatic change in the wake of the end-Permian biotic crisis. Geology 35, 291-294.

Gavrilova V.A.1980. Nekotorye pozdneolenekskie ammonoidei Gornogo Mangyšlaka. (Some late Olenekian ammonoids of Gornyj Mangyshlak.) Ezhegodnik Vsesoyuznogo Paleontologicheskogo Obschestva 23, 16-27.

Gavrilova V.A. 1989. O nekotoryh dinsridah (ammonoidei) Mangyšlaka. (On some dinaritid ammonoids of Mangyshlak.) Ezhegodnik Vsesoyuznogo Paleontologicheskogo Obschestva 32, 162-181.

Griesbach C.L. 1880. Palaeontological notes on the Lower Trias of the Himalayas. Geological Survey of India Records 13(2), 83-113.

Guex J. 1978. Le Trias inférieur des Salt Range (Pakistan): problemes biochronologiques. (Lower Triassic of the Salt Range [Pakistan]: biochronological problems.) Eclogae Geologicae Helvetiae 71, 105-141. 
Hallam A. 1994. The earliest Triassic as anoxic event, and its relationship to the end-Palaeozoic mass extinction. In A.F. Embry et al. (eds.): Pangea: global environments and resources. Pp. 797-804. Calgary: Canadian Society of Petroleum Geologists.

Henderson C. \& Baud A. 1997. Correlation of the Permian-Triassic boundary in Arctic Canada and comparison with Meishan, China. In W. Naiwen \& J. Remane (eds.): Proceedings of the 30th International Geological Congress. Vol. 11. Stratigraphy. Pp. 143-152. Utrecht: VSP.

Horacek M. \& Abart R.B.R. 2007. Carbon isotope record of the P/T boundary and Lower Triassic in the southern Alps: evidence for rapid changes in storage of organic carbon. Palaeogeography, Palaeoclimatology, Palaeoecology 252, 347-354.

Hyatt A. \& Smith J.P. 1905. The Triassic cephalopod genera of America. Professional Paper, Series C, 40. Washington, D.C.: United States Geological Survey.

Jin Y., Shen S., Zhu Z., Mei S. \& Wang W. 1996. The Selong section, candidate of the Global Stratotype Section and Point of the Permian-Triassic boundary. In H. Yin (ed.): The Palaeozoic-Mesozoic boundary. Candidates of the Global Stratotype Section and Point of the Permian-Triassic boundary. Pp. 127-137. Wuhan: China University of Geosciences Press.

Keyserling A.G. 1845. Beschreibung einiger von Dr. A. Th. v. Middendorff miitgebrachten Ceratiten des arctischen Sibiriens. (Description of some ceratites collected by Dr A. Th. v. Middendorff in Arctic Siberia.) Bulletin de la Classe Physico-Mathématique de l'Académie Impériale des Sciences de Saint-Pétersbourg 5(11), 161-174. St. Petersburg: Academy of Sciences.

Kidder D.L. \& Worsley T.R. 2004. Causes and consequences of extreme Permo-Triassic warming to globally equable climate and relation to the Permo-Triassic extinction and recovery. Palaeogeography, Palaeoclimatology, Palaeoecology 203, 207-237.

Kiparisova L.D. 1961. Paleontologičeskoe obosnovanie stratigrafii triasovyh otloženij Primorskogo kraja. 1. Golovonogie molljuski. (Palaeontological basis of Triassic stratigraphy of the Primorye region. 1. Cephalopods.) Trudy Vsesojuznogo Naučnoissledovatel'skogo Geologičeskogo Instituta (VSEGEI), New Series 48. Leningrad: A.P. Karpinsky Russian Geological Research Institute.

Kiparisova L.D. 1972. Paleontologičeskoe obosnovanie stratigrafii triasovyh otloženij Primorskogo kraja. 2. Pozdnetriasovye dvustvorčatye molljuski i obščaja stratigrafija. (Palaeontological basis of Triassic stratigraphy of the Primorye region. 2. Late Triassic molluscs and general stratigraphy.) Trudy Vsesojuznogo Naučno-issledovatel'skogo Geologičeskogo Instituta (VSEGEI), New Series 181. Leningrad: A.P. Karpinsky Russian Geological Research Institute.

Korčinskaja [Korchinskaya] M.V. 1970. Olenekskie ammonity Špicbergena. (Olenekian ammonoids of Spitsbergen.) Utchenyje Zapiski Naučno-issledovatel'skogo Instituta Geologii Arktiki (NIIGA). Paleontologija i Biostratigrafija 27, 80-89.
Korčinskaja [Korchinskaya] M.V. \& Vavilov M.N. 1987. Ranneindskie ammonity Špicbergena (Early Induan ammonoids of Spitsbergen.) In Ju.D. Zaharov \& Ju.I. Onoprienko (eds.): Problemy biostratigrafii permi i triasa Vostoka SSSR. (Problems of Permian and Triassic biostratigraphy of the East USSR.) Pp. 64-73. Vladivostok: Dalnevostočnyj Naučnyj Centr Akademii Nauk SSSR.

Korte C., Jasper T., Kozur H.W. \& Veizer J. 2005. $\delta^{18} \mathrm{O}$ and $\delta^{13} \mathrm{C}$ of Permian brachiopods: a record of seawater evolution and continental glaciation. Palaeogeography, Palaeoclimatology, Palaeoecology 224, 333-351.

Korte C., Kozur H.W. \& Veizer J. 2005. $\delta^{18} \mathrm{O}$ and $\delta^{13} \mathrm{C}$ values of Triassic brachiopods and carbonate rocks as proxies for coeval seawater and palaeotemperature. Palaeogeography, Palaeoclimatology, Palaeoecology 226, 287-306.

Kotljar [Kotlyar] G.V., Zaharov Ju.D. [Zakharov Y.D.], Kočirkevič B.V., Kropačeva G.S., Rostovcev K.O., Cedija I.O., Vuks G.P. \& Guseva E.A. 1983. Pozdnepermskij etap $v$ evolucii organičeskogo mira. Džulfinskij i Dorašamskij jarusy SSSR. (Evolution of the Latest Permian biota. Dzhulfian and Dorashamian regional stages in the USSR.) Leningrad: Nauka.

Kotlyar G.V., Belyansky G.S., Burago V.I., Nikitina A.P., Zakharov Y.D. \& Zhuravlev A.V. 2006. South Primorye, Far East-a key region for global Permian correlation. Journal of Asian Earth Sciences 26, 280-293.

Kotlyar G.V., Zakharov Y.D. \& Polubotko I.V. 2004. Late Changhsingian fauna of the northwestern Caucasus mountains, Russia. Journal of Paleontology 78, 513-527.

Kozur H.W. 1998. Problems for evaluation of the scenario of the Permian-Triassic boundary biotic crisis and its causes. Geologia Croatica 51, 135-162.

Kozur H.W. 2004. Pelagic uppermost Permian and Permian-Triassic boundary conodonts of Iran. Part 1. Taxonomy. Hallesches Jahrbuch für Geowissenschaften, Series B 18, 39-68.

Kozur H.W. 2007. Biostratigraphy and event stratigraphy in Iran around the Permian-Triassic Boundary (PTB): implications for the causes of the PTB biotic crisis. Global and Planetary Change 55, 155-176.

Kozur H.W., Ramovs A., Wang C. \& Zakharov Y.D. 1995. The importance of Hindeodus parvus (Conodonta) for the definition of the Permian-Triassic boundary and evaluation of the proposed section for a Global Stratotype Section and Point (GSSP) for the base of the Triassic. Geologija 37, 173-213.

Krull E.S., Lehrmann D.J., Druke D., Kessel B., Yu Y.Y. \& Li R. 2004. Stable carbon isotope stratigraphy across the Permian-Triassic boundary in shallow marine carbonate platforms, Nanpanjian Basin, south China. Palaeogeography, Palaeoclimatology, Palaeoecology 204, 297-315.

Krystyn L. 1974. Die Tirolites-Fauna (Ammonoidea) der untertriadischen Werfener Schichten Europas und ihre stratigraphische Bedeutung. (Tirolites fauna of the Lower Triassic Werfen Shale in Europe and their stratigraphical significance.) Sitzungsberichte der Österreichische Akademie der Wissenschaften, Mathematisch-Naturwissenschaftliche Klasse, Abteilung I, 183, 29-50. Vienna. 
Krystyn L. \& Orchard M.J. 1996. Lowermost Triassic ammonoid and conodont biostratigraphy of Spiti, India. Albertiana 17, 10-21.

Kummel B. 1965. New Lower Triassic ammonoids from New Zealand. New Zealand Journal of Geology and Geophysics 8, 537-547.

Kummel B. 1969. Ammonoids of the Late Scythian (Lower Triassic). Bulletin of the Museum of Comparative Zoology 137, 311-701.

Kummel B. 1972. The Lower Triassic (Scythian) ammonoid Otoceras. Bulletin of the Museum of Comparative Zoology 143, 365-418.

Kurušin [Kurushin] N.I. \& Zaharov [Zakharov] V.A. 1995. Klimat severnoj Sibiri v triasovyj period. (Climate in north Siberia through the Triassic period.) Bulleten Moskovskogo Obschestva Ispytatelei Prirody, Serija Geologičeskaja 70, $55-60$.

Lazurkin D.V. \& Korčinskaja [Korchinskaya] M.V. 1963. O stratotipe olenekskogo jarusa. (The stratotype of the Olenekian Stage.) Trudy Naučno-issledovatel'skogo Instituta Geologii Arktiki (NIIGA) 136, 99-104.

Lehrmann D., Ramezani J., Bowring S.A., Martin M.W., Montgomery P., Enos P., Payne J.L., Orchard M.J., Wang H. \& Wei J. 2006. Timing of recovery from the end-Permian extinction: geochronologic and biostratigraphic constraints from south China. Geology 34, 1053-1056.

Leonova T.B. 2002. Permian ammonoids: classification and phylogeny. Paleontological Journal 36, Supplement 1. Moscow: Nauka.

Magaritz M. 1989. ${ }^{13} \mathrm{C}$ minima follow extinction events: a clue to faunal radiation. Geology 17, 337-340.

Martin E.E. \& Macdougall J.D. 1995. Sr and Nd isotopes at the Permian/Triassic boundary: a record of climate change. Chemical Geology 125, 73-99.

McGowan A. 2005. Ammonoid recovery from the Late Permian mass extinction event. Comptes Rendus Paleovol 4, 517-530.

Mei S. \& Henderson C.M. 2001. Evolution of Permian conodont provincialism and its significance in global correlation and paleoclimate implication. Palaeogeography, Palaeoclimatology, Palaeoecology 170, 237-260.

Mei S., Zhang K. \& Wardlaw B.R. 1998. A refined succession of Changhsingian and Griesbachian neogondolellid conodonts from the Meishan section, candidate of the global stratotype section and point of the Permian-Triassic boundary. Palaeogeography, Palaeoclimatology, Palaeoeology 143, 213-226.

Menning M., Ogg J.G. \& Romer R.L. 2007. Calibration of early and Middle Triassic time-scale using orbital-climate cycles. New Mexico Museum of Natural History and Science Bulletin 41, 273-274.

Mojsisovics E. 1886. Arctische Triasfaunen. Beiträge zur paläontologischen Charakteristik der Arktisch-Pacifischen Triasprovinz. (Arctic Triassic fauna. Contribution to palaeontological characteristics of the Arctic-Pacific Triassic province.) Mémoires de l'Académie Impériale des Sciences de
St.-Pétersbourg, Series 7, 33(6). St. Petersburg: Academy of Sciences.

Mojsisovics E., Waagen W. \& Diener C. 1895. Entwurf einer Gliederung der pelagischen Sedimente des Trias-Systems. (Draft division of pelagic sediments of the Triassic system.) Sitzungsberichte der Kaiserlichen Akademie der Wissenschaften Wien 104(1), 1271-1302. Vienna.

Mørk A., Elvebakk G., Forsberg A.W., Hounslow M.W., Nakrem H.A.,Vigran J.O. \& Weitschat W. 1999. The type section of the Vikinghøgda Formation: a new Lower Triassic unit in central and eastern Svalbard. Polar Research $18,51-82$.

Mundil R., Ludwiq K.R., Metcalfe I \& Renne P.R. 2004. Age and timing of the Permian mass extinction: $\mathrm{U} / \mathrm{Pb}$ dating of closed-system zircons. Science 305, 1760-1776.

Musashi M., Isozaki Y., Koike T. \& Kreulen R. 2001. Stable carbon isotope signature in mid-Panthalassa shallow-water carbonates across the Permo-Triassic boundary: evidence for ${ }^{13} \mathrm{C}$-depleted superocean. Earth and Planetary Science Letters 191, 9-20.

Nakazawa K. 1981. Analysis of the Late Permian and Early Triassic faunas of Kashmir. Memoirs of the Geological Survey of India 46, 191-204.

Nakazawa K. 1993. Stratigraphy of the Permian-Triassic transition and the Paleozoic-Mesozoic boundary. Bulletin of the Geological Survey in Japan 44, 425-445.

Nakazawa K. \& Kapoor H.M. (eds.) 1981. The Upper Permian and Lower Triassic faunas of Kashmir. Palaeontologia Indica, New Series 45. Calcutta.

Nassichuk W.W. 1995. Permian ammonoids in the Arctic regions of the world. In P.A. Schholle et al. (eds.): The Permian of Northern Pangea. Vol. 1. Paleogeography, paleoclimates, stratigraphy. Pp. 210-235. Berlin: Springer.

Nassichuk W.W., Furnish W.M. \& Glenister B.F. 1966. The Permian ammonoids of Arctic Canada. Geological Survey of Canada Bulletin 131. Ottawa: Geological Survey of Canada.

Okuneva T.M. 1976. Rannetriasovye molljuski hrebta Bol'šie Curki. (Early Triassic molluscs from the Bolshiye Churki Range region [Khabarovsk area].) Trudy Vsesojuznogo Naučno-issledovatel'skogo Geologičeskogo Instituta (VSEGEI), New Series 263, 28-45.

Orchard M.J. \& Krystyn L. 1998. Conodonts of the lowermost Triassic of Spiti, and new zonation based on Neogondolella succession. Rivista Italiana di Paleontologia e Stratigrafia 10, 341-368.

Orchard M.J. \& Tozer E.T. 1997. Triassic conodont biochronology, its calibration with the ammonoid standard, and a biostratigraphic summery for the western Canada sedimentary basin. Bulletin of Canadian Petroleum Geologists 45, 675-692.

Ovtcharova M., Bucher H., Schaltegger U., Galfetti T., Brayard A. \& Guex J. 2006. New Early to Middle Triassic $\mathrm{U}-\mathrm{Pb}$ ages from South China calibration with ammonoid biochronozones and implications for the timing of the Triassic recovery. Earth and Planetary Science Letters 243, 463-475. 
Payne J.L. \& Kump L.R. 2007. Evidence for recurrent Early Triassic massive volcanism from quantitative interpretation of carbon isotope fluctuations. Earth and Planetary Science Letters 256, 264-277.

Payne J.L., Lehrmann D.J., Wei J., Orchard M.J., Schrag D.P. \& Knoll A.H. 2004. Large perturbations of the carbon cycle during recovery from the end-Permian extinction. Science 305, 506-509.

Perri M.C. \& Farabegoli E. 2003. Conodonts across the Permian-Triassic boundary in the Southern Alps. Courier Forschugsinstitut Senckenberg 245, 281-313.

Popov Ju.N. [Popow Y.N.] 1939. Triasovye otloženija v rajone istokov r. Kolymy. (Triassic sediments in the Kolyma waterhead.) Problemy Arktiki 12, 83-86.

Popov Ju.N. [Popow Y.N.] 1958. Nahodka Otoceras v nižnem triase Vostočnogo Verhojan'ja. (A find of Otoceras in the Lower Triassic of the eastern Verkhoyansk region.) Izvestiya Akademii Nauk SSSR, Serija Geologicheskaja 2, 105-109.

Popov Ju.N. [Popow N.] 1968. Rannetriasovye ammonoidei zony Prohungarites similis. (Early Triassic ammonoids from the Prohungarites similis Zone of the north Yakut area.) Paleontologicheskij Žurnal 3, 134-137.

Popov Ju.N. [Popow Y.N.] 1970. Ammonoidei. (Ammonoids.) Trudy Naučno-issledovatel'skogo Instituta Geologii Arktiki (NIIGA) 154, 113-140.

Riccardi A., Kump L.R., Arthur M.A. \& D’Hondt S. 2007. Carbon isotopic evidence for chemocline upward excursions during the end-Permian event. Palaeogeography, Palaeoclimatology, Palaeoecology 248, 73-81.

Richoz S. 2006. Stratigraphie et variations isotopiques du carbone dans le permien supérieur et le trias inférieur de quelgues localités de la Néotéthys (Turquie, Oman et Iran). (Stratigraphy and variation of carbon isotope values in some Upper Permian and Lower Triassic localites of the Neotethys [Turkey, Oman and Iran].) Mémoires de Géologie 46. Lausanne: Earth Sciences Dept., Lausanne University.

Ševyrev [Shevyrev] A.A. 1968. Triasovye ammonoidei juga SSSR. (Triassic ammonoids of the south USSR.) Trudy Paleontologicheskogo Instituta Akademii Nauk SSSR 119. Moscow: Nauka.

Ševyrev [Shevyrev] A.A. 1999. Nižnjaja granica triasa iee korreljacija v morskih otloženijah. 1. Pograničnye razrezy $\mathrm{v}$ Tetise. (Lower boundary of the Triassic and its correlation within marine sediments. 1. Near boundary sections in the Tethys.) Stratigrafiya, Geologicheskaya Korrelyatsiya 7, 14-27.

Ševyrev [Shevyrev] A.A. 1990. Ammonoidei i čronostratigrafija triasa. (Ammonoids and chronostratigraphy of the Triassic.) Trudy Paleontologicheskogo Instituta Akademii Nauk SSSR 24. Moscow: Nauka.

Ševyrev [Shevyrev] A.A. 2000. Nižnjaja granica triasa iee korreljacija v morskih otloženijah. 2. Boreal'nye rzrezy bazal'nogo triasa i ih korreljacija s pograničnymi razrezami Tetisa. (Lower boundary of the Triassic and its correlation within marine sediments. 2. Boreal sections of the basal Triassic and their correlation with near boundary sections of the Tethys.) Stratigrafiya, Geologicheskaya Korrelyatsiya 8, 55-65.

Ševyrev [Shevyrev] A.A. 2002. Ammonitovye zony olenekskogo jarusa (nižnij trias) i ih korreljacija. (Ammonite zones of the Olenekian stage [Lower Triassic] and their correlation.) Stratigrafiya, Geologicheskaya Korrelyatsiya 10, 59-69.

Shen S.Z., Archbold N.W. \& Shi G.R. 2000. Changhsingian (Late Permian) brachiopod palaeobiogeoraphy. Historical Biology 15, 121-134.

Shen S.Z., Cao C.Q., Henderson C.M., Wang X.-D., Shi G.R., Wang Y. \& Wang W. 2006. End-Permian mass extinction pattern in the northern peri-Gondwanan region. Palaeoworld 15, 3-30.

Shi G.R. 2006. The marine Permian of east and northeast Asia: an overview of biostratigraphy, palaeobiogeography and palaeogeographical implications. Journal of Asian Earth Sciences 26, 175-206.

Spath L.F. 1930. The Eotriassic invertebrate fauna of East Greenland. Meddelelser om Gronland 83. Copenhagen: Commission for Scientific Research in Greenland.

Tong J.N., Zakharov Y.D. \& Wu S.B. 2004. Early Triassic ammonoid succession in Chaohu, Anhui Province. Acta Palaeontologica 43, 192-204.

Tozer E.T. 1994. Canadian Triassic ammonoid fauna. Geological Survey of Canada Bulletin 467. Ottawa: Geological Survey of Canada.

Tozer E.T. \& Parker J.R. 1968. Notes on the Triassic biostratigraphy of Svalbard. Geological Magazine 105, 526-542.

Yin H. \& Zhang K. 1996. Eventostratigraphy of the Permian-Triassic boundary at Meishan section, South China. In H. Yin (ed.): The Palaeozoic-Mesozoic boundary candidates of the Global Stratotype Section and Point of the Permian-Triassic boundary. Pp. 84-96. Wuhan: China University of Geosciences Press.

Yin H., Zhang K., Wu Sh. \& Peng Y. 1996. Global correlation and definition of the Permian-Triassic boundary. In H. Yin (ed.): The Palaeozoic-Mesozoic boundary candidates of the Global Stratotype Section and Point of the Permian-Triassic boundary. Pp. 3-28. Wuhan: China University Geosciences Press.

Voinova E.F., Kiparisova L.D. \& Robinson V.N. 1947. Klass Cephalopoda. Golovonogie. (Class Cephalopoda.) In L.D. Kiparisova (ed.): Atlas rukovodjaščih iskopaemuh faun SSSR. 7. Triasovaja sistema. (Atlas of the guide forms of the fossil faunas of the USSR. 7. Triassic system.) Pp. 124-176. Leningrad: Gosgeolizdat.

Vuks V.Y. 1997. Triassic foraminifers of Russia and adjacent countries (Caucasus, Mangyshlak, Pamirs). In C.A. Ross et al. (eds.): Late Paleozoic foraminifera, their biostratigraphy, evolution, and paleoecology; and the mid-Carboniferous boundary. Special Publication 36. Pp. 159-162. Washington, D.C.: Cushman Foundation for Foraminiferal Research.

Wang Y. 1985. Remarks on the Scythian-Anisian boundary. Rivista Italiana di Paleonologia e Stratigrafia 90, 515-544.

Welter O.A. 1915. Die Ammoniten und Nautiliden der ladinischen und anisischen Trias von Timor. (Ammonoids 
and nautilids from the Ladinian and Anisian Triassic of Timor.) Palaontologie von Timor 5, 71-136.

Wignall P.B., Kozur H. \& Hallam A. 1996. On the timing of paleoenvironmental changes at the Permo-Triassic $(\mathrm{P} / \mathrm{Tr})$ boundary using conodont biostratigraphy. Historical Biology $12,39-62$.

Wignall P.B. \& Twitchett R.J. 2002. Permian-Triassic sedimentology of Jameson Land, East Greenland: incised submarine channels in an anoxic basin. Journal of the Geological Society 159, 691-703.

Worsley D. 2006. The post-Caledonian geological development of Svalbard and the Barents Sea. Boreal Triassic 2006. Longyearbyen, Svalbard, 16-19 August 2006. NGF Abstracts and Proceedings of the Geological Society of Norway 3, $5-22$.

Xu G. \& Liu G. 1983. Some problems in the research of Triassic brachiopods. In Yang Z. et al. (eds.): Triassic of the South Qilian Mountains. Pp. 67-83. Beijing: Geological Publishing House. (In Chinese.)

Zaharov Ju.D. [Zakharov Y.D.] 1968. Biostratigrafija $i$ ammonoidei nižnego triasa Južnogo Primor'ja. (Biostratigraphy and ammonoids of the Lower Triasic of South Primorye.) Moscow: Nauka.

Zaharov Ju.D. [Zakharov Y.D.] 1971. Otoceras Boreal'noj provincii. (Otoceras of the Boreal province.) Paleonologičeskij Žurnal 3, 50-59.

Zaharov Ju.D. [Zakharov Y.D.] 1977. The main features of geographical differentiation of marine invertebrates in the Early Triassic. V.A. Krasilov (ed.): Evoljucija organičeskogo mira Tihookeanskogo pojasa. Hronologičeskie i paleobiogeograficheskie rubeži. (Organic evolution in the circum-Pacific belt. Chronological and paleobiogeographical boundaries.) Pp. 63-88. Vladivostok: Dalnevostočnyj Naučnyj Centr Akademii Nauk SSSR.

Zaharov Ju.D. [Zakharov Y.D.] 1978. Rannetriasovye ammonoidei Vostoka SSSR. (Lower Triassic ammonoids of the USSR.) Moscow: Nauka.

Zaharov Ju.D. [Zakharov Y.D.] 1983. Novye permskie ciclolobidy (Goniatitida) juga SSSR. (New Permian cyclolobids [Goniatitida] of the south USSR.) Paleontologičeskij Žurnal 2, 26-130.

Zaharov Ju.D. [Zakharov Y.D.] 1985. K voprosu o tipe granicy permi i triasa. (On the type of the Permian-Triassic boundary.) Bjulleten' Moskovskogo Obšcestva Ispytatelej Prirody, Serija Geologičeskaja 60, 59-70.

Zaharov Ju.D. [Zakharov Y.D.], Boriskina N.G. \& Popov A.M. 2001. Rekonstrukcija uslovij morskoj sredy pozdnego paleozoja $i$ mezozoja po izotopnym dannym (na primere severa Evrazii). (The reconstruction of Late Paleozoic and Mesozoic marine environments from isotopic data [evidence from north Eurasia].) Vladivostok: Dalnauka.

Zaharov Ju.D. [Zakharov Y.D.] \& Pavlov A.M. 1986a. Pervaja nahodka araksoceratid $\mathrm{v}$ permi vostočnoj časti SSSR. (The first find of araxoceratid ammonoids in the Permian of the eastern USSR.) In Ju.D. Zaharov \& Ju.I. Onoprienko (eds.): Permo-Triasovye sobytija $v$ razvitii organičeskogo mira Severo-Vostočcnoj Azii. (Permian-Triassic events during evolution of the north-east Asia biota.) Pp. 74-85. Vladivostok: Dalnevostočnyj Naučnyj Centr Akademii Nauk SSSR.

Zaharov Ju.D. [Zakharov Y.D.] \& Pavlov A.M. 1986b. Permskie cefalopody Primor'ja i problema zonal'nogo rasčlenenija permi Tetičeskoj oblasti. (Permian cephalopods of Primorye and the problem of the Permian zonal stratification in the Tethys.) In Ju.D. Zaharov \& Ju.I. Onoprienko (eds.): Korreljacija permo-triasovyh otloženij vostoka SSSR. (Correlation of Permo-Triassic sediments of the east USSR.) Pp. 5-32. Vladivostok: Dalnevostočnyj Naučnyj Centr Akademii Nauk SSSR.

Zaharov Ju.D. [Zakharov Y.D.], Popov A.M., Šorohova [Shorochova] S.A. \& Volynec [Volynets] E.B. 2008. In P.V. Markevič \& Ju.D. Zaharov (eds.): Trias i jura Sihote-Alinja. 2. Vulkanogenno-osadočnyj kompleks, paleobiogeografija. (Triassic and Jurassic of the Sikhote-Alin. 2. Volcano-sedimentory assemblage, paleobiogeography.) Pp. 232-239. Vladivostok: Dalnauka.

Zaharov Ju.D. [Zakharov Y.D.] \& Sokarev A.N. 1991a. Permian-Triassic paleomagnetism of Eurasia. In T. Kotaka et al. (eds.): Proceedings of the International Symposium on Shallow Tethys 3, Sendai, Japan, 20-23 September 1990. Pp. 313-323. Sendai: The Saito Gratitude Foundation. Zaharov Ju.D. [Zakharov Y.D.] \& Sokarev A.N. 1991b. Biostratigrafija i paleomagnetizm permi i triasa Evrazii (Biostratigraphy and palaeomagnetism of the Permian of Eurasia.) Moscow: Nauka.

Zakharov Y.D. 1974. The importance of palaeobiogeographical data for the solution of the problem on the Lower Triassic division. Schriftenreihe der Erdwissenschaftlichen Kommissionen 2, 237-243.

Zakharov Y.D. 1983. Palaeosuccession and basic factors of syngenetic during the time of the Permian-Triassic boundary. Sitzungsberichte der Österreichische Akademie der Wissenschaften, Mathematisch-naturwissenschaftliche Klasse, Abteilung I, 192, 37-58.

Zakharov Y.D. 1986. Type and hypotype of the Permian-Triassic boundary. Memorie della Societa Geologica Italiana 34, 277-289.

Zakharov Y.D. 1992. The Permo-Triassic boundary in the southern and eastern USSR and its intercontinental correlation. In W.C. Sweet et al. (eds.): Permo-Triassic events in the eastern Tethys. Stratigraphy, classification and relations with the western Tethys. World and Regional Geology 2. Pp. 46-55. Cambridge: Cambridge University Press.

Zakharov Y.D. 1996. The Induan-Olenekian boundary in the Tethys and Boreal realm. Annali dei Musei civici di Rovereto, Sezione: Archeologia, Storia e Scienze Naturali 11, 133-156.

Zakharov Y.D. 1997. Ammonoid evolution and the problem of the stage and substage division of the Lower Triassic. Mémoires de Géologie (Lausanne) 30, 121-132.

Zakharov Y.D. 2002. Ammonoid succession of the Setorym River (Verkhoyansk area) and the problem of the Permian-Triassic boundary in Boreal realm. Journal of China University of Geosciences 13, 107-123. 
Zakharov Y.D. 2007. Examples of Late Olenekian invertebrate successions. Paper 2. Arctic Siberia (Mengilyakh Creek). Albertiana 35, 52-58.

Zakharov Y.D., Biakov A.S., Baud A. \& Kozur H. 2005. Significance of Caucasian sections for working out carbon-isotope standard for Upper Permian and Lower Triassic (Induan) and their correlation with the Permian of north-eastern Russia. Journal of China University of Geosciences 16, 141-151.

Zakharov Y.D., Boriskina N.G., Cherbadzhi A.K., Popov A.M. \& Kotlyar G.V. 1999. Main trends in Permo-Triassic shallow-water temperature changes: evidence from the oxygen isotope and $\mathrm{Ca}-\mathrm{Mg}$ ratio data. Albertiana 23, $11-22$.

Zakharov Y.D. \& Ehiro M. in press. New data on Guadalupian and earliest Lopingian Timorites of Far East (Japan and Russia) and biostratigraphical and paleogeographical significance of cyclolobid ammonoids. In K. Tanabe $\&$ H. Hirano (eds.): Proceedings of the 7 th International Symposium, Cephalopods-Present and Past. Tokyo: Tokai University Press.

Zakharov Y.D. \& Oleinikov A.V. 1994. New data on the problem of the Permian-Triassic boundary in the Far East.
In A.F. Embry et al. (eds.): Pangea: global environments and resources. Pp. 845-856. Calgary: Canadian Society of Petroleum Geologists.

Zakharov Y.D. \& Popov A.M. 2007. Examples of Late Olenekian invertebrate successions. Paper 1. Western Tethys (Dolnapa, Mangyshlak). Albertiana 35, 46-51.

Zakharov Y.D., Popov A.M. \& Buryi G.I. 2005. Unique marine Olenekian-Anisian boundary section from South Primorye, Russian Far East. Journal of China University of Geosciences 16, 419-230.

Zakharov Y.D., Ukhaneva N.G., Ignatiev A.V., Afanasyeva T.B., Buryi G.I., Panasenko E.S., Popov A.M., Punina T.A. \& Cherbadzhi A.K. 2000. Latest Permian and Triassic carbonates of Russia: new palaeontological findings, stable isotopes, Ca-Mg ratio and correlation. In H. Yin et al. (eds.): Permian-Triassic evolution of Tethys and western circum-Pacific developments in paleontology and stratigraphy. Pp. 141-171. Amsterdam: Elsevier.

Ziegler A.M., Gibbs M.T. \& Hulver M.L. 1999. A mini-atlas of oceanic water in the Permian period. Proceedings of the Royal Society of Victoria 110, 323-344. 